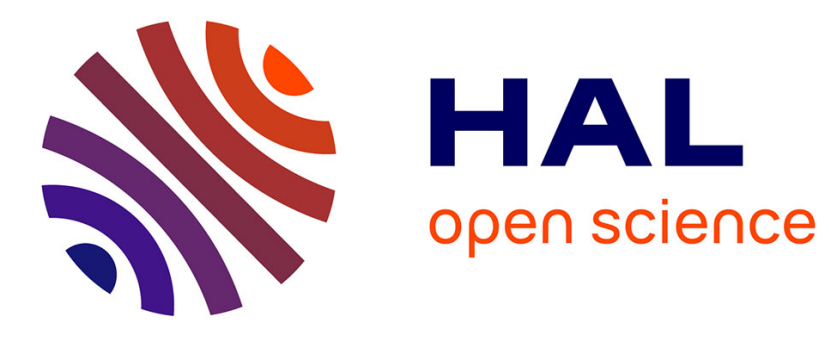

\title{
Infinitesimal deformations of Levi flat hypersurfaces
}

Andrei Iordan

\section{To cite this version:}

Andrei Iordan. Infinitesimal deformations of Levi flat hypersurfaces. Bollettino dell'Unione Matematica Italiana, In press, 10.1007/s40574-018-0176-y . hal-01897005

\section{HAL Id: hal-01897005 https://hal.sorbonne-universite.fr/hal-01897005}

Submitted on 16 Oct 2018

HAL is a multi-disciplinary open access archive for the deposit and dissemination of scientific research documents, whether they are published or not. The documents may come from teaching and research institutions in France or abroad, or from public or private research centers.
L'archive ouverte pluridisciplinaire HAL, est destinée au dépôt et à la diffusion de documents scientifiques de niveau recherche, publiés ou non, émanant des établissements d'enseignement et de recherche français ou étrangers, des laboratoires publics ou privés. 


\title{
Infinitesimal deformations of Levi flat hypersurfaces
}

\author{
Andrei lordan ${ }^{1}$
}

In 2009, at the Conference of Complex Analysis and Geometry-Levico Terme, Paolo de Bartolomeis proposed me to study the deformations of Levi flat hypersurfaces. From 2010, we met several months a year and wrote several papers on this subject. In the first semester of 2016, I visited the Laboratory Fibonacci in Pisa and we had a lot of interesting discussions about the connection between the classical paper of Kodaira and Spencer, where they studied the foliations by means of the DGLA of graded derivations, and our papers. The present paper has its roots in these discussions.

We planned to continue our work in Paris during the spring 2017, but unfortunately, Paolo passed away on 29th of November 2016. The mathematical research of Paolo holds a major position in the great tradition of Italian geometers. I am honoured to have been Paolo's collaborator and friend.

\begin{abstract}
In order to study the deformations of foliations of codimension 1 of a smooth manifold $L$, de Bartolomeis and Iordan defined the DGLA $\mathcal{Z}^{*}(L)$, where $\mathcal{Z}^{*}(L)$ is a subset of differential forms on $L$. In another paper, de Bartolomeis and Iordan studied the deformations of foliations of a smooth manifold $L$ by defining the canonical solutions of Maurer-Cartan equation in the DGLA of graded derivations $\mathcal{D}^{*}(L)$. Let $L$ be a Levi flat hypersurface in a complex manifold. Then the deformation theories in $\mathcal{Z}^{*}(L)$ and $\mathcal{D}^{*}(L)$ lead to the moduli space for the Levi flat deformations of $L$. In this paper we discuss the relationship between the infinitesimal deformations of $L$ defined by the solutions of Maurer-Cartan equation in $\mathcal{Z}^{*}(L)$ and the infinitesimal deformations of $L$ obtained by means of the canonical solutions of MaurerCartan equation in the DGLA of graded derivations $\mathcal{D}^{*}(L)$.
\end{abstract}

Keywords Levi flat hypersurface · Differential graded Lie Algebras · Maurer-Cartan equation · Foliations · Graded derivations

Mathematics Subject Classification Primary 32G10 · 32E99 · 16W25 · 53C12

\section{Introduction}

In [7] Kodaira and Spencer's studied the deformations of multifoliate structures by means of the DGLA of graded derivations whose underlying graded algebra was defined by Frölicher and Nijenhuis in [4]. They defined as in [6,9] the infinitesimal deformations and proved that

Andrei Iordan

andrei.iordan@imj-prg.fr

1 Institut de Mathématiques de Jussieu-Paris Rive Gauche, Faculté des Sciences et Ingénierie (former Université Pierre et Marie Curie), Sorbonne Université, 4 Place Jussieu, 75252 Paris Cedex 05, France 
the infinitesimal deformations are represented by the tangent vectors at origin of the solutions of Maurer Cartan equation in the DGLA of graded derivations.

In the paper [1], de Bartolomeis and the author studied deformations of Levi flat hypersurfaces in complex manifolds. For this purpose, we elaborated a theory of deformations of integrable distributions of codimension 1 in smooth manifolds. Our approach was different from [7] (see remark 14 of [1] for a discussion).

In [1], the DGLA algebra $\left(\mathcal{Z}^{*}(L), \delta,\{\cdot, \cdot\}\right)$ associated to a codimension 1 co-orientable foliation on a manifold $L$ is a subalgebra of the the algebra $\left(\Lambda^{*}(L), \delta,\{\cdot, \cdot\}\right)$ of differential forms on $L$. Its definition depends on the choice of a DGLA defining couple $(\gamma, X)$, where $\gamma$ is a 1-differential form on $L$ and $X$ is a vector field on $L$ such that $\gamma(X)=1$, but the cohomology classes of the underlying differential vector space structure do not depend on its choice. The deformations are given by forms $\alpha \in \mathcal{Z}^{1}(L)$ verifying the MaurerCartan equation $\delta \alpha+\frac{1}{2}\{\alpha, \alpha\}=0$ and the moduli space takes in account the diffeomorphic deformations.

The infinitesimal deformations along curves are subsets of of the first cohomology group of the DGLA $\left(\mathcal{Z}^{*}(L), \delta,\{\cdot, \cdot\}\right)$, which are represented by tangent vectors at the origin of curves with values in $\mathfrak{M C}_{\delta}(L)=\left\{\alpha \in \mathcal{Z}^{1}(L): \delta \alpha+\frac{1}{2}\{\alpha, \alpha\}=0\right\}$.

If $L$ is a Levi flat hypersurface in a complex manifold, we defined the canonical DGLA defining couple for the Levi foliation of $L$. Then we parametrized the Levi flat hypersurfaces near $L$ by means of the set of smooth functions on $L$ and we obtained a partial differential equation for the infinitesimal Levi flat deformations.

In [2] we defined canonical solutions of the Maurer-Cartan equation in the DGLA of graded derivations by means of deformations of the $d$-operator depending on a vector valued differential 1-form $\Phi$ and we gave a classification of these solutions depending on their type. A canonical solution $e_{\Phi}$ of the Maurer-Cartan equation associated to an endomorphism $\Phi$ is of finite type $r$ if there exists $r \in \mathbb{N}$ such that $\Phi^{r}[\Phi, \Phi]_{\mathcal{F N}}=0$ and $r$ is minimal with this property, where $[\cdot, \cdot]_{\mathcal{F} \mathcal{N}}$ is the Frölicher-Nijenhuis bracket. We proved that a distribution $\xi$ of codimension $k$ on a smooth manifold is integrable if and only if the canonical solution of the Maurer-Cartan equation associated to the endomorphism of the tangent space which is the trivial extension of the $k$-identity on a complement of $\xi$ in $T M$ is of finite type $\leqslant 1$. In [3] we proved that the deformation theory in the DGLA of graded derivation is not obstructed, but it is level-wise obstructed.

Let $L$ be a smooth Levi flat hypersurface in a complex manifold and consider a Levi flat deformation $\left(L_{a_{t}}\right)$ of $L$ given by a family $\left(a_{t}\right)$ of smooth functions on $L$. Let $\left(\omega_{a(t)}, Y_{a(t)}\right)$ be the canonical DGLA defining couple of $L_{a_{t}}$ and $e_{\omega_{a(t)} \otimes Y_{a(t)}}$ the canonical solution of the Maurer-Cartan equation in the DGLA of graded derivations associated to $\omega_{a(t)} \otimes Y_{a(t)}$. The family of 1 -forms $\left(\omega_{a(t)}\right)$ are the inverse images of a family of 1-forms on 1-forms $\left(\gamma_{a(t)}\right)$ on $L$. In this paper we show that the infinitesimal deformation represented by the tangent at the origin to the curve $t \mapsto \alpha_{a_{t}}$, where $\left(\alpha_{a_{t}}\right)$ are solutions of Maurer-Cartan equation in the DGLA $\left(\mathcal{Z}^{*}(L), \delta,\{\cdot, \cdot\}\right)$, is also represented by the tangent at the origin to the curve $t \mapsto \gamma_{a(t)}$.

\section{Preliminaries}

\subsection{The DGLA of graded derivations}

In this subsection we recall some definitions and properties of the DGLA of graded derivations from [4,7] (see, also [8]). 
Definition 1 A differential graded Lie agebra (DGLA) is a triple $\left(V^{*}, d,[\cdot, \cdot]\right)$ such that:

1. $V^{*}=\oplus_{i \in \mathbb{N}} V^{i}$, where $\left(V^{i}\right)_{i \in \mathbb{N}}$ is a family of $\mathbb{C}$-vector spaces and $d: V^{*} \rightarrow V^{*}$ is a graded homomorphism such that $d^{2}=0$. An element $a \in V^{k}$ is said to be homogeneous of degree $k=\operatorname{deg} a$.

2. $[\cdot, \cdot]: V^{*} \times V^{*} \rightarrow V^{*}$ defines a structure of graded Lie algebra i.e., for homogeneous elements we have

$$
[a, b]=-(-1)^{\operatorname{deg} a \operatorname{deg} b}[b, a]
$$

and

$$
[a,[b, c]]=[[a, b], c]+(-1)^{\operatorname{deg} a \operatorname{deg} b}[b,[a, c]]
$$

3. $d$ is compatible with the graded Lie algebra structure i.e.,

$$
d[a, b]=[d a, b]+(-1)^{\operatorname{deg} a}[a, d b] .
$$

Definition 2 Let $\left(V^{*}, d,[\cdot, \cdot]\right)$ be a DGLA and $a \in V^{1}$. We say that $a$ verifies the MaurerCartan equation in $\left(V^{*}, d,[\cdot, \cdot]\right)$ if

$$
d a+\frac{1}{2}[a, a]=0 .
$$

Definition 3 Let $A=\oplus_{k \in \mathbb{Z}} A_{k}$ be a graded algebra. A linear mapping $D: A \rightarrow A$ is called a graded derivation of degree $p=|D|$ if $D: A_{k} \rightarrow A_{k+p}$ and $D(a b)=D(a) b+$ $(-1)^{p \operatorname{deg} a} a D(b)$.

Notation 1 Let $M$ be a smooth manifold. We denote by $\Lambda^{*} M$ the algebra of differential forms on $M$, by $\mathfrak{X}(M)$ the Lie algebra of vector fields on $M$ and by $\Lambda^{*} M \otimes T M$ the algebra of $T M$-valued differential form on $M$, where $T M$ is the tangent bundle to $M$. In the sequel, we will identify $\Lambda^{1} M \otimes T M$ with the algebra End $(T M)$ of endomorphisms of $T M$ by their canonical isomorphism: for $\sigma \in \Lambda^{1} M, X, Y \in \mathfrak{X}(M),(\sigma \otimes X)(Y)=\sigma(Y) X$.

Definition 4 Let $M$ be a smooth manifold. We denote by $\mathcal{D}^{*}(M)$ the graded algebra of graded derivations of $\Lambda^{*} M$.

Definition 5 Let $P, Q$ be homogeneous elements of degree $|P|,|Q|$ of $\mathcal{D}^{*}(M)$. We define

$$
\begin{gathered}
{[P, Q]=P Q-(-1)^{|P||Q|} Q P,} \\
\neg P=[d, P] .
\end{gathered}
$$

Lemma 1 Let $M$ be a smooth manifold. Then $\left(\mathcal{D}^{*}(M),[\cdot, \cdot], 7\right)$ is a DGLA.

Definition 6 Let $\alpha \in \Lambda^{*} M$ and $X \in \mathfrak{X}(M)$. We define $\mathcal{L}_{\alpha \otimes X}, \mathcal{I}_{\alpha \otimes X}$ by

$$
\begin{aligned}
\mathcal{L}_{\alpha \otimes X} \sigma= & \alpha \wedge \mathcal{L}_{X} \sigma+(-1)^{|\alpha|} d \alpha \wedge \iota_{X} \sigma, \sigma \in \Lambda^{*}(M) \\
& \mathcal{I}_{\alpha \otimes X} \sigma=\alpha \wedge \iota_{X} \sigma, \sigma \in \Lambda^{*}(M),
\end{aligned}
$$

where $\mathcal{L}_{X}$ is the Lie derivative and $\iota_{X}$ the contraction by $X$.

For $\Phi \in \Lambda^{*} M \otimes T M$ we define $\mathcal{L}_{\Phi}, \mathcal{I}_{\Phi}$ as the extensions by linearity of (2.1), (2.2).

Lemma 2 For every $D \in \mathcal{D}^{k}(M)$ there exist unique forms $\Phi \in \Lambda^{k} M \otimes T M, \Psi \in \Lambda^{k+1} M \otimes$ $T M$ such that

$$
D=\mathcal{L}_{\Phi}+\mathcal{I}_{\Psi}
$$

Denote $\mathcal{L}(\mathcal{D})=\mathcal{L}_{\Phi}$ and $\mathcal{I}(\mathcal{D})=\mathcal{I}_{\psi}$. 
It is proved in [7], Theorem 5.3, that the solutions of Maurer-Cartan equation in the DGLA of graded derivations define a deformation of a foliated manifold and an infinitesimal foliation is a class in the cohomology group $H^{1}(M, \Theta)$, where $\Theta$ is the sheaf associated to the multifoliate structure; moreover, the infinitesimal deformation is represented by the tangent vector at the origin to a curve with values in the set of solutions of Maurer-Cartan equation in the DGLA of graded derivations.

\subsection{Canonical solutions of Maurer-Cartan equation in the algebra of graded derivations}

This subsection gives some definitions and results from [2].

Definition 7 Let $\Phi \in \Lambda^{1} M \otimes T M$.

(a) Let $\sigma \in \Lambda^{p} M$. We define $\Phi \sigma \in \Lambda^{p} M$ by $\Phi \sigma=\sigma$ if $p=0$ and

$$
(\Phi \sigma)\left(V_{1}, \ldots, V_{p}\right)=\sigma\left(\Phi V_{1}, \ldots, \Phi V_{p}\right) \text { if } p \geqslant 1, V_{1}, \ldots, V_{p} \in \mathfrak{X}(M) .
$$

(b) Let $\Psi \in \Lambda^{p} M \otimes T M$. We define $\Phi \Psi \in \Lambda^{p} M \otimes T M$ by $\Phi \Psi=\Psi$ if $p=0$ and

$$
\Phi \Psi\left(V_{1}, \ldots, V_{p}\right)=\Phi\left(\Psi\left(V_{1}, \ldots, V_{p}\right)\right), V_{1}, \ldots, V_{p} \in \mathfrak{X}(M) \text { if } p \geqslant 1 .
$$

Theorem 1 Let $\Phi \in \Lambda^{1} M \otimes T M$ such that $R_{\Phi}=I d_{T M}+\Phi$ is invertible. Set

$$
e_{\Phi}=R_{\Phi} d R_{\Phi}^{-1}-d
$$

Then:

(i) $e_{\Phi}$ is a solution of the Maurer-Cartan equation in $\left.\left(\mathcal{D}^{*}(M),\right\urcorner,[\cdot, \cdot]\right)$.

(ii) Let $D$ be a solution of the Maurer-Cartan equation in $\left(\mathcal{D}^{*}(M), 7,[\cdot, \cdot]\right)$. Let $\Phi \in$ $\Lambda^{1} M \otimes T M$ such that $\mathcal{L}(D)=\mathcal{L}_{\Phi}$. Suppose that $R_{\Phi}=I d_{T(M)}+\Phi$ is invertible. Then $D=e_{\Phi}$.

Definition 8 Let $\Phi \in \Lambda^{1} M \otimes T M$ such that $I d_{T(M)}+\Phi$ is invertible. $e_{\Phi}$ is called the canonical solution of Maurer-Cartan equation associated to $\Phi$.

\subsection{Canonical DGLA defining couple for a Levi flat hypersurface}

In this subsection we will use the following setting and notations from [1]:

Let $L$ be a $C^{\infty}$ manifold and $\xi \subset T(L)$ an integrable distribution of codimension 1 . We denote by $\Lambda^{k}(L)$ the $k$-forms on $L$ and $\Lambda^{*}(L)=\oplus_{k \in \mathbb{N}} \Lambda^{k}(L)$. For $\alpha, \beta \in \Lambda^{*}(L)$, $X \in \mathfrak{X}(L)$, set

$$
\{\alpha, \beta\}=\mathcal{L}_{X} \alpha \wedge \beta-\alpha \wedge \mathcal{L}_{X} \beta
$$

where $\mathcal{L}_{X}$ is the Lie derivative.

Let $\gamma \in \wedge^{1}(L)$ such that $\operatorname{ker} \gamma=\xi$ and $X$ a vector field on $L$ such that $\gamma(X)=1$. Set

$$
\delta=d_{\gamma}=d+\{\gamma, \cdot\}
$$

and

$$
\mathcal{Z}^{*}(L)=\left\{\alpha \in \Lambda^{*}(L): \iota_{X} \alpha=0\right\}
$$

Then $\left(\mathcal{Z}^{*}(L), \delta,\{\cdot, \cdot\}\right)$ is a DGLA. 
Remark 1 Let $L$ be a $C^{\infty}$ manifold and $\xi \subset T(L)$ an integrable distribution of codimension 1. Then there exists a 1 -form $\gamma$ on $L$ such that $\xi=\operatorname{ker} \gamma$ if and only if $\xi$ is co-orientable, i.e., the normal space to the foliation defined by $\xi$ is orientable (see, for ex. [5]).

Definition 9 Let $L$ be a $C^{\infty}$ manifold and $\xi \subset T(L)$ an integrable co-orientable distribution of codimension 1. A couple $(\gamma, X)$ where $\gamma \in \wedge^{1}(L)$ and $X$ is a vector field on $L$ such that ker $\gamma=\xi$ and $\gamma(X)=1$ will be called a DGLA defining couple.

We denote

$$
\mathfrak{M C}_{\delta}(L)=\left\{\alpha \in \mathcal{Z}^{1}(L): \delta a+\frac{1}{2}\{\alpha, \alpha\}=0\right\}
$$

the set of solutions of Maurer-Cartan equation in $\left(\mathcal{Z}^{*}(L), \delta,\{\cdot, \cdot\}\right)$.

It is proved in [1] that the moduli space of deformations of integrable distributions of codimension 1 is represented in $H^{1}\left(\mathcal{Z}^{*}(L), \delta\right)$ by the tangent vectors at 0 to $\mathfrak{M C}_{\delta}(L)$ valued curves.

Definition 10 The infinitesimal deformations of $L$ in $\left(\mathcal{Z}^{*}(L), \delta,\{\cdot, \cdot\}\right)$ are the collection of cohomology classes in $H^{1}\left(\mathcal{Z}^{*}(L), \delta\right)$ of the tangent vectors at 0 to $\mathfrak{M C}_{\delta}(L)$ valued curves.

In the sequel we will use the following setting and notations:

Let $M$ be a complex manifold and $L$ a Levi flat hypersurface of class $C^{k}$ in $M, k \geqslant 2$; then there exists $r \in C^{k}(M), d r \neq 0$ on $L$ such that $L=\{z \in M: r(z)=0\}$ and set $j: L \rightarrow M$ the natural inclusion. As $d r \neq 0$ on a neighborhood of $L$ in $M$ we will suppose in the sequel that $d r \neq 0$ on $M$.

We denote by $J$ the complex structure on $M$ and let $\gamma=j^{*}\left(d_{J}^{c} r\right), d_{J}^{c} r=-J d r$. The Levi distribution $T(L) \cap J T(L)=$ ker $\gamma$ is integrable.

Let $g$ be a fixed Hermitian metric on $M$ and $Z=\operatorname{grad}_{g} r /\left\|\operatorname{grad}_{g} r\right\|_{g}^{2}$. Then the vector field $X=J Z$ is tangent to $L$ and verifies

$$
\gamma(X)=d_{J}^{c} r(J Z)=1 .
$$

Definition 11 For a given defining function $r$ and a Hermitian metric on $M$, we will fix $\gamma$ and $X$ defined before and we will call $(\gamma, X)$ the canonical DGLA defining couple associated to the Levi foliation on $L$.

\subsection{Deformations of Levi flat hypersurfaces}

As in [1], we will give a parametrization of real hypersurfaces near a given hypersurface $L$ and diffeomorphic to $L$ as graphs over $L$ :

Let $U$ be a tubular neighborhood of $L$ in $M$ and $\pi: U \rightarrow L$ the projection on $L$ along the integral curves of $Z$. As we are interested in infinitesimal deformations we suppose $U=M$.

Let $a \in C^{\infty}(L ; \mathbb{R})$. Denote

$$
L_{a}=\{z \in M: r(z)=a(\pi(z))\} .
$$

Since $Z$ is transverse to $L, L_{a}$ is a hypersurface in $M$. Consider the map $\Phi_{a}: M \rightarrow M$ defined by $\Phi_{a}(p)=q$, where

$$
\pi(q)=\pi(p), r(q)=r(p)+a(\pi(p)) .
$$

In particular, for every $x \in L$ we have

$$
r\left(\Phi_{a}(x)\right)=a(x) .
$$


$\Phi_{a}$ is a diffeomorphism of $M$ such that $\Phi_{a}(L)=L_{a}$ and $\Phi_{a}^{-1}=\left.\pi\right|_{L_{a}}$.

Conversely, let $\Psi \in \mathcal{U} \subset \operatorname{Diff}(M)$, where $\mathcal{U}$ is a suitable neighborhood of the identity in Diff $(M)$. Then there exists $a \in C^{\infty}(L)$ such that $\Psi(L)=L_{a}$. Indeed, for $x \in L$, let $q(x) \in \Psi(L)$ such that $\pi(q(x))=x$. By defining $a(x)=r(q(x))$, we obtain $\Psi(L)=L_{a}$.

So we have the following:

Lemma 3 Let $\Psi \in \mathcal{U}$. There exists a unique a $\in C^{\infty}(L)$ such that $\Psi(L)=L_{a}$.

It follows that a neighborhood $\mathcal{V}$ of 0 in $C^{\infty}(L)$ is a set of parametrization of hypersurfaces close to $L$.

For $a \in \mathcal{V}$, consider the almost complex structure $J_{a}=\left(\Phi_{a}^{-1}\right)_{*} \circ J \circ\left(\Phi_{a}\right)_{*}$ on $M$ and denote

$$
\alpha_{a}=\left(d_{J_{a}}^{c} r(X)\right)^{-1} j^{*}\left(d_{J_{a}}^{c} r\right)-\gamma .
$$

Then $\alpha_{a} \in \mathcal{Z}^{1}(L)$ and we have the following

Proposition 1 For every $a \in \mathcal{V}$, the following assertions are equivalent:

(i) $L_{a}$ is Levi flat.

(ii) $\alpha_{a}$ satisfies the Maurer-Cartan equation in $\left(\mathcal{Z}^{*}(L), \delta,\{\cdot, \cdot\}\right)$ i.e.,

$$
\delta \alpha_{a}+\frac{1}{2}\left\{\alpha_{a}, \alpha_{a}\right\}=0 .
$$

Definition 12 A 1-dimensional Levi flat deformation of $L$ is a smooth mapping $\Psi: I \times M \rightarrow$ $M$ such that $\Psi_{t}=\Psi(t, \cdot) \in \operatorname{Diff}(M), L_{t}=\Psi_{t} L$ is a Levi flat hypersurface in $M$ for every $t \in I$ and $L_{0}=L$.

By Proposition 1 a Levi flat deformation of $L$ is given by a family $\left(a_{t}\right)_{t \in I}$ in $\mathcal{V}$ such that the associated family $\left(\alpha_{a_{t}}\right)_{t \in I}$ of 1 -forms satisfies the Maurer-Cartan equation (2.6) in $\left(\mathcal{Z}^{*}(L), \delta,\{\cdot, \cdot\}\right)$ for every $t$.

Set $j_{a_{t}}: L_{a_{t}} \rightarrow M$ the natural embedding, $r_{a_{t}}=r-a_{t} \circ \pi$ and $\eta_{a_{t}}=j_{a_{t}}^{*}\left(d^{c} r_{a_{t}}\right) \in$ $\Lambda^{1}\left(L_{a_{t}}\right)$. Let $\left(\eta_{a_{t}}, Y_{a_{t}}\right)$ be the canonical defining couple for the Levi flat hypersurface $L_{a_{t}}$ associated to $r_{a_{t}}$ and set $\sigma(t)=\eta_{a_{t}} \otimes Y_{a_{t}}$. Then $e_{\sigma(t)}$ is the canonical solution of MaurerCartan equation in the algebra of graded derivations and the tangent vector to the curve $t \mapsto \sigma(t)$ represents the infinitesimal deformation. By setting $\gamma_{a_{t}}=\Phi_{a_{t}}^{*}\left(\eta_{a_{t}}\right) \in \Lambda^{1}(L)$ and $X_{a_{t}}=\left(\Phi_{a_{t}}^{-1}\right)_{*}\left(Y_{a_{t}}\right) \in \mathcal{H}(L)$, we have $\gamma_{a_{t}}\left(X_{a_{t}}\right)=1$. Since ker $\eta_{a_{t}}$ is integrable if and only if ker $\gamma_{a_{t}}$ is integrable, $\left(\gamma_{a_{t}}, X_{a_{t}}\right)$ is a DGLA defining couple for the integrable distribution ker $\gamma_{a_{t}} \subset T L$.

\section{Infinitesimal deformations}

Recall that in the sequel we use the setting and the notations of the previous section: for a given Levi flat hypersurface $L$ in a complex manifold $M$, we will fix a defining function $r$, a Hermitian metric $g$ on $M$, the canonical DGLA defining couple $(\gamma, X)$ for the Levi foliation associated to $r$; also, for $a \in C^{\infty}(L)$, we will use the meaning of $L_{a}, \Phi_{a}, j_{a}, r_{a}, \eta_{a}, \gamma_{a}, \alpha_{a}$, defined before.

An equivalent form of Proposition 5 of [1] is the following:

Proposition 2 Let $L$ be a Levi flat hypersurface in a complex manifold $M,\left(a_{t}\right)_{t \in I}$ a family in $C^{\infty}(L)$ defining a Levi flat deformation of $L$ and $p=\frac{d a_{t}}{d t}{ }_{\mid t=0}$. Then for every vector field 
$V$ in a neighborhood of $L$ such that the restriction of $V$ to $L$ is a section of $T L \cap J T L$ we have

$$
{\frac{d \alpha_{a_{t}}}{d t}{ }_{\mid t=0}}(V)=p \iota_{X} J d d^{c} r(V)-d^{c} p(V)=-\delta^{c} p .
$$

where $\delta^{c}: \mathcal{Z}^{*}(L) \rightarrow \mathcal{Z}^{*}(L)$ is defined for $\alpha \in \mathcal{Z}^{p}(L)$ and $V_{1}, \ldots, V_{p+1} \in T(L) \cap J T(L)$ by

$$
\delta^{c} \alpha\left(V_{1}, \ldots, V_{p+1}\right)=J^{-1} \delta J \alpha\left(V_{1}, \ldots, V_{p+1}\right) .
$$

We will give firstly a different proof of Proposition 2 by using the following Lemma:

Lemma 4 Let $L$ be a Levi flat hypersurface in a complex manifold $M$ and $\left(a_{t}\right)_{t \in I}$ a family of smooth functions on $L$ defining a Levi flat deformation of $L$. Then for every vector field $V$ in a neighborhood of $L$ such that the restriction of $V$ to $L$ is a section of $T L \cap J T L$ we have

$$
\begin{aligned}
\left(\Phi_{a_{t}}\right)_{*, \Phi_{a_{t}}(\eta)}^{-1} J\left(\Phi_{a_{t}}\right)_{*, \eta}(V)= & J V(\eta)+t(p \circ \pi)(\eta)([Z, J V](\eta)-J[Z, V](\eta)) \\
& -t\left(\left(p_{*}\right)_{\pi\left(\Phi_{a_{t}}(\eta)\right)}(J V)\right) Z(\eta) \\
& +t\left(\left(p_{*}\right)_{\pi\left(\Phi_{a_{t}}(\eta)\right)}(V)\right)(J Z) \eta+o(t) .
\end{aligned}
$$

Proof Step 1. First order development of $\Phi_{a_{t}}$ and $\left(\Phi_{a_{t}}\right)_{*}$ by means of coordinates $\eta$ such that $Z=\frac{\partial}{\partial \eta_{1}}$.

Let $x \in L$ and consider holomorphic coordinates $z=\left(z_{1}, \ldots, z_{n}\right)$ in a neighborhood $U$ of $x$ in $M, z_{j}=x_{j}+i y_{j}, j=1, \ldots, n$. Denote by $\xi=\left(\xi_{1}, \ldots, \xi_{2 n}\right)$ the corresponding real coordinates, $\xi_{2 j-1}=x_{j}, \xi_{2 j}=y_{j}, j=1, \ldots, n$.

We may suppose that $Z_{1}(x) \neq 0$ and consider a smooth change of coordinates $\xi=\xi(\eta)$ in a neighborhood of $x$ such that $Z=\frac{\partial}{\partial \eta_{1}}$ defined by:

$$
\begin{aligned}
Z & =\sum_{j=1}^{2 n} Z_{j}^{\xi} \frac{\partial}{\partial \xi_{j}}=\sum_{j=1}^{2 n} Z_{j}^{\eta} \frac{\partial}{\partial \eta_{j}}=\sum_{j=1}^{2 n} Z_{j}^{\xi} \sum_{i=1}^{2 n} \frac{\partial \eta_{i}}{\partial \xi_{j}} \frac{\partial}{\partial \eta_{i}} \\
& =\sum_{i=1}^{2 n}\left(\sum_{j=1}^{2 n} Z_{j}^{\xi} \frac{\partial \eta_{i}}{\partial \xi_{j}}\right) \frac{\partial}{\partial \eta_{i}}=\frac{\partial}{\partial \eta_{1}},
\end{aligned}
$$

so

$$
\sum_{j=1}^{2 n} Z_{j}^{\xi} \frac{\partial \eta_{1}}{\partial \xi_{j}}=1, \sum_{j=1}^{2 n} Z_{j}^{\xi} \frac{\partial \eta_{i}}{\partial \xi_{j}}=0, i \geqslant 2
$$

which means

$$
\frac{\partial(\eta)}{\partial(\xi)} Z^{\xi}=(1,0, \ldots, 0) \Longleftrightarrow Z^{\xi}=\left(\frac{\partial(\eta)}{\partial(\xi)}\right)^{-1} e_{1},
$$

where $e_{1}=(1,0, \ldots, 0)$.

Denote $\left(\frac{\partial(\eta)}{\partial(\xi)}\right)^{-1}=\left(a_{i j}\right)_{1 \leqslant i, j \leqslant 2 n}$ and we choose

$$
a_{i 1}=Z_{i}^{\xi}, i=1, \ldots, n, a_{i j}=\delta_{i}^{j}, \quad i=1, \ldots, n, j=2, \ldots, n
$$

where $\delta_{i}^{j}=1$ if $i=j, \delta_{i}^{j}=0$ otherwise. 
Let $V$ be a vector field on $M$ in a neighborhood of $x, V(\eta)=\sum_{j=1}^{2 n} a_{j}(\eta)\left(\frac{\partial}{\partial \eta_{j}}\right)_{\eta}$. Since for every $j=1, \ldots, 2 n$ we have

$$
\left(\frac{\partial}{\partial \eta_{j}}\right)_{\eta}=\sum_{j=1}^{2 n} \frac{\partial \xi_{j}}{\partial \eta_{i}}(\eta)\left(\frac{\partial}{\partial \xi_{j}}\right)_{\xi(\eta)}=\sum_{i=1}^{n} \frac{\partial x_{i}}{\partial \eta_{j}}(\eta)\left(\frac{\partial}{\partial x_{i}}\right)_{\xi(\eta)}+\sum_{i=1}^{n} \frac{\partial y_{i}}{\partial \eta_{j}}(\eta)\left(\frac{\partial}{\partial y_{i}}\right)_{\xi(\eta)}
$$

and

$$
J\left(\frac{\partial}{\partial \eta_{j}}\right)_{\eta}=\sum_{i=1}^{n}-\frac{\partial y_{i}}{\partial \eta_{j}}(\eta)\left(\frac{\partial}{\partial x_{i}}\right)_{\xi(\eta)}+\sum_{i=1}^{n} \frac{\partial x_{i}}{\partial \eta_{j}}(\eta)\left(\frac{\partial}{\partial y_{i}}\right)_{\xi(\eta)}
$$

it follows that

$$
\begin{aligned}
J V(\eta)=\sum_{j=1}^{2 n} a_{j}(\eta) J\left(\frac{\partial}{\partial \eta_{j}}\right)_{\eta}= & \sum_{k=1}^{2 n}\left(\sum_{j=1}^{2 n} a_{j}(\eta) \sum_{i=1}^{n}-\frac{\partial y_{i}}{\partial \eta_{j}}(\eta) \frac{\partial \eta_{k}}{\partial x_{i}}(\xi(\eta))\right. \\
& \left.+\frac{\partial x_{i}}{\partial \eta_{j}}(\eta) \frac{\partial \eta_{k}}{\partial y_{i}}(\xi(\eta))\right)\left(\frac{\partial}{\partial \eta_{k}}\right)_{\eta}
\end{aligned}
$$

We will consider $t$ small enough in order that $\Phi_{a_{t}}(U) \subset U$.

Suppose that in a neighborhood of $x$ the hypersuface $L$ is given by

$$
L=\left\{\eta_{1}=\varphi\left(\eta^{\prime}\right)\right\}
$$

where

$$
\eta^{\prime}=\left(\eta_{2}, \ldots, \eta_{n}\right) .
$$

Since the flow of $Z$ is $F(\eta, t)=\left(\eta_{1}+t, \eta^{\prime}\right), \pi$ is given by

$$
\pi(\eta)=\left(\varphi\left(\eta^{\prime}\right), \eta^{\prime}\right)
$$

and

$$
\Phi_{a_{t}}(\eta)=\Phi_{a_{t}}\left(\eta_{1}, \eta^{\prime}\right)=\left(\eta_{1}+\lambda_{t}(\eta), \eta^{\prime}\right),
$$

where $\lambda_{t}$ is a smooth real function on a neighborhood of $x$ verifying

$$
r\left(\eta_{1}+\lambda_{t}(\eta), \eta^{\prime}\right)=r(\eta)+a_{t}\left(\varphi\left(\eta^{\prime}\right), \eta^{\prime}\right),
$$

and $\lambda_{0}=0$.

From (3.8) it follows that

$$
\frac{d}{d t}\left(r\left(\eta_{1}+\lambda_{t}(\eta), \eta^{\prime}\right)\right)=\frac{\partial r}{\partial \eta_{1}}\left(\eta_{1}+\lambda_{t}(\eta), \eta^{\prime}\right) \frac{d \lambda_{t}}{d t}(\eta)=\frac{d}{d t}\left(a_{t}\left(\varphi\left(\eta^{\prime}\right), \eta^{\prime}\right)\right),
$$

which can be written as

$$
\frac{\partial r}{\partial \eta_{1}}\left(\eta_{1}+\lambda_{t}(\eta), \eta^{\prime}\right) \frac{d \lambda_{t}}{d t}(\eta)=\frac{d}{d t} a_{t}(\pi(\eta)) .
$$

For $t=0$ we obtain

$$
\frac{\partial r}{\partial \eta_{1}}(\eta) \frac{d \lambda_{t}}{d t}(\eta)_{\mid t=0}=p(\pi(\eta))
$$


So

$$
\begin{aligned}
{\frac{d \lambda_{t}}{d t}{ }_{\mid t=0}}^{=} & \frac{1}{\frac{\partial r}{\partial \eta_{1}}}(p \circ \pi)=\frac{1}{Z(r)}(p \circ \pi)=\frac{1}{d r(Z)}(p \circ \pi) \\
= & \frac{1}{d r\left(\frac{\text { gradg }_{g} r}{\left\|\operatorname{grad}_{g} r\right\|^{2}}\right)}(p \circ \pi)=p \circ \pi
\end{aligned}
$$

and

$$
\lambda_{t}(\eta)=t p(\pi(\eta))+o(t) .
$$

It follows that

$$
\Phi_{a_{t}}(\eta)=\eta+\left(\lambda_{t}(\eta), 0, \ldots, 0\right)=\eta+t p(\pi(\eta), 0, \ldots, 0)+o(t),
$$

so

$$
\begin{aligned}
\frac{\partial \Phi_{a_{t}}^{1}}{\partial \eta_{1}} & =1+t \frac{\partial(p \circ \pi)}{\partial \eta_{1}}+o(t), \frac{\partial \Phi_{a_{t}}^{1}}{\partial \eta_{k}}=t \frac{\partial(p \circ \pi)}{\partial \eta_{k}}+o(t), k \geqslant 2, \\
\frac{\partial \Phi_{a_{t}}^{j}}{\partial \eta_{k}} & =\delta_{k}^{j}+o(t), j \geqslant 2, k \geqslant 1,
\end{aligned}
$$

where $\delta_{k}^{j}=0$ if $j \neq k$ and $\delta_{k}^{j}=1$ if $j=k$.

Analoguously we obtain

$$
\begin{aligned}
\Phi_{a_{t}}^{-1}(\eta) & =\left(\eta_{1}-\lambda_{t}(\eta), \eta^{\prime}\right)=\eta-t p(\pi(\eta)) e_{1}+o(t), \\
\frac{\partial\left(\Phi_{a_{t}}^{-1}\right)^{1}}{\partial \eta_{1}} & =1-t \frac{\partial(p \circ \pi)}{\partial \eta_{1}}+o(t), \frac{\partial\left(\Phi_{a_{t}}^{-1}\right)^{1}}{\partial \eta_{k}}=-t \frac{\partial(p \circ \pi)}{\partial \eta_{k}}+o(t), k \geqslant 2, \\
\frac{\partial\left(\Phi_{a_{t}}^{-1}\right)^{j}}{\partial \eta_{k}} & =\delta_{k}^{j}+o(t), j, k \geqslant 2 .
\end{aligned}
$$

Step 2. First order development of $\left(\Phi_{a_{t}}\right)_{*, \Phi_{a_{t}}(\eta)}^{-1} J\left(\Phi_{a_{t}}\right)_{*, \eta}(V)$.

Let $V$ be a vector field on $M$ in a neighborhood of $x, V(\eta)=\left(\sum_{i=1}^{2 n} a_{i}(\eta)\left(\frac{\partial}{\partial \eta_{i}}\right)_{\eta}\right)$.

By (3.10) we have

$$
\begin{aligned}
\left(\Phi_{a_{t}}\right)_{*, \eta}(V)= & \sum_{j=1}^{2 n}\left(\sum_{k=1}^{2 n} \frac{\partial \Phi_{a_{t}}^{j}}{\partial \eta_{k}}(\eta) a_{k}(\eta)\right)\left(\frac{\partial}{\partial \eta_{j}}\right)_{\Phi_{a_{t}}(\eta)} \\
= & \left(\left(1+t \frac{\partial(p \circ \pi)}{\partial \eta_{1}}(\eta)\right) a_{1}(\eta)+t \sum_{k=2}^{2 n} \frac{\partial(p \circ \pi)}{\partial \eta_{k}}(\eta) a_{k}(\eta)\right)\left(\frac{\partial}{\partial \eta_{1}}\right)_{\Phi_{a_{t}}(\eta)} \\
& +\sum_{j=2}^{2 n} \sum_{k=1}^{2 n} \delta_{j}^{k} a_{k}(\eta)\left(\frac{\partial}{\partial \eta_{j}}\right)_{\Phi_{a_{t}}(\eta)}+o(t) \\
= & \sum_{j=1}^{2 n} a_{j}(\eta)\left(\frac{\partial}{\partial \eta_{j}}\right)_{\Phi_{a_{t}}(\eta)}+t \sum_{k=1}^{2 n} \frac{\partial(p \circ \pi)}{\partial \eta_{k}} a_{k}(\eta)\left(\frac{\partial}{\partial \eta_{1}}\right)_{\Phi_{a_{t}}(\eta)}+o(t)
\end{aligned}
$$


and by using (3.4) we obtain

$$
\begin{aligned}
J\left(\Phi_{a_{t}}\right)_{*, \eta}(V)= & \sum_{j=1}^{2 n} a_{j}(\eta) J\left(\frac{\partial}{\partial \eta_{j}}\right)_{\Phi_{a_{t}}(\eta)} \\
& +t(p \circ \pi)_{* \eta}\left(\sum_{j=1}^{2 n} a_{j}(\eta)\left(\frac{\partial}{\partial \eta_{j}}\right)\right) J\left(\frac{\partial}{\partial \eta_{1}}\right)_{\Phi_{a_{t}}(\eta)}+o(t) \\
= & \sum_{j=1}^{2 n} a_{j}(\eta) \sum_{k=1}^{2 n} \sum_{i=1}^{n}-\frac{\partial y_{i}}{\partial \eta_{j}}\left(\Phi_{a_{t}}(\eta)\right) \frac{\partial \eta_{k}}{\partial x_{i}}\left(\xi\left(\Phi_{a_{t}}(\eta)\right)\right)\left(\frac{\partial}{\partial \eta_{k}}\right)_{\Phi_{a_{t}}(\eta)} \\
& +\sum_{j=1}^{2 n} a_{j}(\eta) \sum_{k=1}^{2 n} \sum_{i=1}^{n} \frac{\partial x_{i}}{\partial \eta_{j}}\left(\left(\Phi_{a_{t}}(\eta)\right)\right) \frac{\partial \eta_{k}}{\partial y_{i}}\left(\xi\left(\Phi_{a_{t}}(\eta)\right)\right)\left(\frac{\partial}{\partial \eta_{k}}\right)_{\Phi_{a_{t}}(\eta)} \\
& +t(p \circ \pi)_{* \eta}\left(\sum_{j=1}^{2 n} a_{j}(\eta)\left(\frac{\partial}{\partial \eta_{j}}\right)\right) J\left(\frac{\partial}{\partial \eta_{1}}\right)_{\Phi_{a_{t}}(\eta)}+o(t) .
\end{aligned}
$$

Analoguosly, for a vector field $\sum_{k=1}^{2 n} b_{k} \frac{\partial}{\partial \eta_{k}}$ on $U$ by (3.11) we have

$$
\begin{aligned}
& \left(\left(\Phi_{a_{t}}\right)_{*}^{-1}\right)_{\Phi_{a_{t}}(\eta)}\left(\sum_{k=1}^{2 n} b_{k}\left(\Phi_{a_{t}}(\eta)\right)\left(\frac{\partial}{\partial \eta_{k}}\right)_{\Phi_{a_{t}}(\eta)}\right) \\
& =\sum_{k=1}^{2 n} b_{k}\left(\Phi_{a_{t}}(\eta)\right)\left(\frac{\partial}{\partial \eta_{k}}\right)_{\eta} \\
& \quad-t(p \circ \pi)_{* \Phi_{a_{t}}(\eta)}\left(\sum_{k=1}^{2 n} b_{k}\left(\Phi_{a_{t}}(\eta)\right)\left(\frac{\partial}{\partial \eta_{k}}\right)_{\Phi_{a_{t}}(\eta)}\right)\left(\frac{\partial}{\partial \eta_{1}}\right)_{\eta}+o(t)
\end{aligned}
$$

and by (3.12) it follows that

$$
\begin{aligned}
& \left(\Phi_{a_{t}}\right)_{* \Phi_{a_{t}}(\eta)}^{-1}\left(J\left(\Phi_{a_{t}}\right)_{* \eta}\left(\sum_{j=1}^{2 n} a_{j}(\eta)\left(\frac{\partial}{\partial \eta_{j}}\right)\right)\right) \\
& =\left(\Phi_{a_{t}}\right)_{* \Phi_{a_{t}}(\eta)}^{-1}\left(\sum_{k=1}^{2 n}\left(\sum_{j=1}^{2 n} a_{j}(\eta)\left(\sum_{i=1}^{n}-\frac{\partial y_{i}}{\partial \eta_{j}}\left(\Phi_{a_{t}}(\eta)\right) \frac{\partial \eta_{k}}{\partial x_{i}}\left(\xi\left(\Phi_{a_{t}}(\eta)\right)\right)\left(\frac{\partial}{\partial \eta_{k}}\right)_{\Phi_{a_{t}}(\eta)}\right)\right)\right) \\
& \quad+\left(\Phi_{a_{t}}\right)_{* \Phi_{a_{t}}(\eta)}^{-1}\left(\sum_{k=1}^{2 n}\left(\sum_{j=1}^{2 n} a_{j}(\eta)\left(\frac{\partial x_{i}}{\partial \eta_{j}}\left(\Phi_{a_{t}}(\eta)\right) \frac{\partial \eta_{k}}{\partial y_{i}}\left(\xi\left(\Phi_{a_{t}}(\eta)\right)\right)\right)\left(\frac{\partial}{\partial \eta_{k}}\right)_{\Phi_{a_{t}}(\eta)}\right)\right) \\
& \quad+t(p \circ \pi)_{* \eta \eta}\left(\sum_{j=1}^{2 n} a_{j}(\eta)\left(\frac{\partial}{\partial \eta_{j}}\right)\right)\left(\Phi_{a_{t}}\right)_{* \Phi_{a_{t}}(\eta)}^{-1}\left(J\left(\frac{\partial}{\partial \eta_{1}}\right)_{\Phi_{a_{t}}(\eta)}\right)
\end{aligned}
$$




$$
\begin{aligned}
= & \sum_{k=1}^{2 n}\left(\sum_{j=1}^{2 n} a_{j}(\eta) \sum_{i=1}^{n}-\frac{\partial y_{i}}{\partial \eta_{j}}\left(\Phi_{a_{t}}(\eta)\right) \frac{\partial \eta_{k}}{\partial x_{i}}\left(\xi\left(\Phi_{a_{t}}(\eta)\right)\right.\right. \\
& \left.+\frac{\partial x_{i}}{\partial \eta_{j}}\left(\Phi_{a_{t}}(\eta)\right) \frac{\partial \eta_{k}}{\partial y_{i}}\left(\xi\left(\Phi_{a_{t}}(\eta)\right)\right)\right)\left(\frac{\partial}{\partial \eta_{k}}\right)_{\eta} \\
& -t(p \circ \pi)_{* \Phi_{a_{t}}(\eta)}\left(J\left(\Phi_{a_{t}}\right)_{* \eta}\left(\sum_{j=1}^{2 n} a_{j}(\eta)\left(\frac{\partial}{\partial \eta_{j}}\right)\right)\right)\left(\frac{\partial}{\partial \eta_{1}}\right)_{\eta} \\
& +t(p \circ \pi)_{* \eta}\left(\sum_{j=1}^{2 n} a_{j}(\eta)\left(\frac{\partial}{\partial \eta_{j}}\right)\right)\left(\Phi_{a_{t}}\right)_{* \Phi_{a_{t}}(\eta)}^{-1}\left(J\left(\frac{\partial}{\partial \eta_{1}}\right)_{\Phi_{a_{t}}(\eta)}\right) \\
= & A+B+C+o(t),
\end{aligned}
$$

where

$$
\begin{aligned}
A= & \sum_{k=1}^{2 n} \sum_{j=1}^{2 n} a_{j}(\eta)\left(\sum _ { i = 1 } ^ { n } \left(-\frac{\partial y_{i}}{\partial \eta_{j}}\left(\Phi_{a_{t}}(\eta)\right) \frac{\partial \eta_{k}}{\partial x_{i}}\left(\xi\left(\Phi_{a_{t}}(\eta)\right)\right)\right.\right. \\
& \left.\left.+\frac{\partial x_{i}}{\partial \eta_{j}}\left(\Phi_{a_{t}}(\eta)\right) \frac{\partial \eta_{k}}{\partial y_{i}}\left(\xi\left(\Phi_{a_{t}}(\eta)\right)\right)\right)\right)\left(\frac{\partial}{\partial \eta_{k}}\right)_{\eta} \\
B= & -t(p \circ \pi)_{* \Phi_{a_{t}}(\eta)}\left(J\left(\Phi_{a_{t}}\right)_{* \eta}\left(\sum_{j=1}^{2 n} a_{j}(\eta)\left(\frac{\partial}{\partial \eta_{j}}\right)\right)\right)\left(\frac{\partial}{\partial \eta_{1}}\right)_{\eta} \\
C= & t(p \circ \pi)_{* \eta}\left(\sum_{j=1}^{2 n} a_{j}(\eta)\left(\frac{\partial}{\partial \eta_{j}}\right)\right)\left(\Phi_{a_{t}}\right)_{* \Phi_{a_{t}}(\eta)}^{-1}\left(J\left(\frac{\partial}{\partial \eta_{1}}\right)_{\Phi_{a_{t}}(\eta)}\right) .
\end{aligned}
$$

Step 3. First order development of $\xi\left(\Phi_{a_{t}}(\eta)\right)$.

By (3.9) it follows that

$$
\xi\left(\Phi_{a_{t}}(\eta)\right)=\xi(\eta)+t(p \circ \pi)(\eta)\left(\frac{\partial \xi^{1}}{\partial \eta_{1}}, \ldots, \frac{\partial \xi^{n}}{\partial \eta_{1}}\right)+o(t) .
$$

By (3.3) we have

$$
\left(\frac{\partial \xi^{1}}{\partial \eta_{1}}, \ldots, \frac{\partial \xi^{n}}{\partial \eta_{1}}\right)=e_{1}
$$

so (3.18) gives

$$
\xi\left(\Phi_{a_{t}}(\eta)\right)=\xi(\eta)+t(p \circ \pi)(\eta) e_{1}+o(t)
$$

and it follows that

$$
\begin{aligned}
\frac{\partial \eta_{k}}{\partial x_{i}}\left(\xi\left(\Phi_{a_{t}}(\eta)\right)\right) & =\frac{\partial \eta_{k}}{\partial x_{i}}\left(\xi(\eta)+t(p \circ \pi)(\eta) e_{1}+o(t)\right) \\
& =\frac{\partial \eta_{k}}{\partial x_{i}}(\xi(\eta))+t(p \circ \pi)(\eta) \frac{\partial}{\partial \eta_{1}}\left(\frac{\partial \eta_{k}}{\partial x_{i}} \xi(\eta)\right)+o(t)
\end{aligned}
$$


and

$$
\begin{aligned}
\frac{\partial \eta_{k}}{\partial y_{i}}\left(\xi\left(\Phi_{a_{t}}(\eta)\right)\right) & =\frac{\partial \eta_{k}}{\partial y_{i}}\left(\xi(\eta)+t(p \circ \pi)(\eta) e_{1}+o(t)\right) \\
& =\frac{\partial \eta_{k}}{\partial y_{i}}(\xi(\eta))+t(p \circ \pi)(\eta) \frac{\partial}{\partial \eta_{1}}\left(\frac{\partial \eta_{k}}{\partial y_{i}} \xi(\eta)\right)+o(t) .
\end{aligned}
$$

In the next steps we will find the first order development of $A, B$ and $C$.

Step 4. First order development of $A$.

By (3.15), (3.9), (3.20) and (3.21) we have

$$
\begin{aligned}
A= & \sum_{k=1}^{2 n} \sum_{j=1}^{2 n} a_{j}(\eta)\left(\sum _ { i = 1 } ^ { n } \left(-\frac{\partial y_{i}}{\partial \eta_{j}}\left(\Phi_{a_{t}}(\eta)\right) \frac{\partial \eta_{k}}{\partial x_{i}}\left(\xi\left(\Phi_{a_{t}}(\eta)\right)\right)\right.\right. \\
& \left.\left.+\frac{\partial x_{i}}{\partial \eta_{j}}\left(\Phi_{a_{t}}(\eta)\right) \frac{\partial \eta_{k}}{\partial y_{i}}\left(\xi\left(\Phi_{a_{t}}(\eta)\right)\right)\right)\right)\left(\frac{\partial}{\partial \eta_{k}}\right) \\
= & \sum_{k=1}^{2 n} \sum_{j=1}^{2 n} a_{j}(\eta)\left(\sum_{i=1}^{n}-\frac{\partial y_{i}}{\partial \eta_{j}}\left(\eta+t(p \circ \pi)(\eta) e_{1}\right)\right)\left(\frac{\partial \eta_{k}}{\partial x_{i}}(\xi(\eta))\right. \\
& \left.+t(p \circ \pi)(\eta) \frac{\partial}{\partial \eta_{1}}\left(\frac{\partial \eta_{k}}{\partial x_{i}} \xi(\eta)\right)\right)\left(\frac{\partial}{\partial \eta_{k}}\right) \\
& +\sum_{k=1}^{2 n} \sum_{j=1}^{2 n} a_{j}(\eta)\left(\sum _ { i = 1 } ^ { n } \frac { \partial x _ { i } } { \partial \eta _ { j } } ( \eta + t ( p \circ \pi ) ( \eta ) e _ { 1 } ) \left(\frac{\partial \eta_{k}}{\partial y_{i}}(\xi(\eta))\right.\right. \\
& \left.\left.+t(p \circ \pi)(\eta) \frac{\partial}{\partial \eta_{1}}\left(\frac{\partial \eta_{k}}{\partial y_{i}} \xi(\eta)\right)\right)\right)\left(\frac{\partial}{\partial \eta_{k}}\right)+o(t) .
\end{aligned}
$$

But

$$
-\frac{\partial y_{i}}{\partial \eta_{j}}\left(\eta+t(p \circ \pi)(\eta) e_{1}\right)=\left(-\frac{\partial y_{i}}{\partial \eta_{j}}(\eta)-t(p \circ \pi)(\eta) \frac{\partial}{\partial \eta_{1}}\left(\frac{\partial y_{i}}{\partial \eta_{j}}\right)(\eta)\right)+o(t),
$$

and

$$
\frac{\partial x_{i}}{\partial \eta_{j}}\left(\eta+t(p \circ \pi)(\eta) e_{1}\right)=\frac{\partial x_{i}}{\partial \eta_{j}}(\eta)+t(p \circ \pi) \frac{\partial}{\partial \eta_{1}}\left(\frac{\partial x_{i}}{\partial \eta_{j}}\right)(\eta)+o(t),
$$

and it follows that

$$
A=A_{1}+t(p \circ \pi)(\eta) A_{2}+t(p \circ \pi)(\eta) A_{3}+o(t),
$$

where

$$
\begin{aligned}
& A_{1}=\sum_{k=1}^{2 n} \sum_{j=1}^{2 n} a_{j}(\eta) \sum_{i=1}^{n}\left(-\frac{\partial y_{i}}{\partial \eta_{j}}(\eta) \frac{\partial \eta_{k}}{\partial y_{i}}(\xi(\eta))+\frac{\partial \eta_{k}}{\partial y_{i}}(\xi(\eta)) \frac{\partial x_{i}}{\partial \eta_{j}}(\eta)\right) \\
& A_{2}=\sum_{k=1}^{2 n}\left(\sum_{j=1}^{2 n} a_{j}(\eta) \sum_{i=1}^{n}\left(-\frac{\partial y_{i}}{\partial \eta_{j}}(\eta)\right) \frac{\partial}{\partial \eta_{1}}\left(\frac{\partial \eta_{k}}{\partial x_{i}} \xi(\eta)\right)+\frac{\partial x_{i}}{\partial \eta_{j}}(\eta) \frac{\partial}{\partial \eta_{1}}\left(\frac{\partial \eta_{k}}{\partial y_{i}} \xi(\eta)\right)\right)\left(\frac{\partial}{\partial \eta_{k}}\right)_{\eta},
\end{aligned}
$$

and

$$
A_{3}=\sum_{k=1}^{2 n} \sum_{j=1}^{2 n} a_{j}(\eta)\left(\sum_{i=1}^{n}-\frac{\partial}{\partial \eta_{1}}\left(\frac{\partial y_{i}}{\partial \eta_{j}}\right)(\eta) \frac{\partial \eta_{k}}{\partial x_{i}}(\xi(\eta))+\frac{\partial}{\partial \eta_{1}}\left(\frac{\partial x_{i}}{\partial \eta_{j}}\right)(\eta) \frac{\partial \eta_{k}}{\partial y_{i}}(\xi(\eta))\right)\left(\frac{\partial}{\partial \eta_{k}}\right) .
$$


From (3.5) we obtain

$$
A_{1}=J V(\eta)
$$

Since

$$
\left(-\frac{\partial y_{i}}{\partial \eta_{j}}(\eta)\right) \frac{\partial}{\partial \eta_{1}}\left(\frac{\partial \eta_{k}}{\partial x_{i}} \xi(\eta)\right)=-\frac{\partial}{\partial \eta_{1}}\left(\frac{\partial y_{i}}{\partial \eta_{j}}(\eta) \frac{\partial \eta_{k}}{\partial x_{i}} \xi(\eta)\right)+\left(\frac{\partial}{\partial \eta_{1}}\left(\frac{\partial y_{i}}{\partial \eta_{j}}(\eta)\right)\right)\left(\frac{\partial \eta_{k}}{\partial x_{i}} \xi(\eta)\right)
$$

and

$$
\frac{\partial x_{i}}{\partial \eta_{j}}(\eta) \frac{\partial}{\partial \eta_{1}}\left(\frac{\partial \eta_{k}}{\partial y_{i}} \xi(\eta)\right)=\frac{\partial}{\partial \eta_{1}}\left(\frac{\partial x_{i}}{\partial \eta_{j}}(\eta) \frac{\partial \eta_{k}}{\partial y_{i}} \xi(\eta)\right)-\frac{\partial}{\partial \eta_{1}}\left(\frac{\partial x_{i}}{\partial \eta_{j}}(\eta)\right)\left(\frac{\partial \eta_{k}}{\partial y_{i}} \xi(\eta)\right),
$$

it follows that

$$
\begin{aligned}
A_{2}= & \sum_{k=1}^{2 n}\left(\sum_{j=1}^{2 n} a_{j}(\eta) \sum_{i=1}^{n}\left(-\frac{\partial}{\partial \eta_{1}}\left(\frac{\partial y_{i}}{\partial \eta_{j}}(\eta) \frac{\partial \eta_{k}}{\partial x_{i}} \xi(\eta)+\frac{\partial}{\partial \eta_{1}}\left(\frac{\partial x_{i}}{\partial \eta_{j}}(\eta) \frac{\partial \eta_{k}}{\partial y_{i}} \xi(\eta)\right)\right)\right)\right)\left(\frac{\partial}{\partial \eta_{k}}\right) \\
& +\sum_{k=1}^{2 n}\left(\sum_{j=1}^{2 n} a_{j}(\eta) \sum_{i=1}^{n} \frac{\partial}{\partial \eta_{1}}\left(\frac{\partial x_{i}}{\partial \eta_{j}}(\eta) \frac{\partial \eta_{k}}{\partial y_{i}} \xi(\eta)\right)-\frac{\partial}{\partial \eta_{1}}\left(\frac{\partial x_{i}}{\partial \eta_{j}}(\eta)\right)\left(\frac{\partial \eta_{k}}{\partial y_{i}} \xi(\eta)\right)\right)\left(\frac{\partial}{\partial \eta_{k}}\right) \\
= & \sum_{k=1}^{2 n}\left(\sum_{j=1}^{2 n} a_{j}(\eta) \sum_{i=1}^{n} \frac{\partial}{\partial \eta_{1}}\left(-\frac{\partial y_{i}}{\partial \eta_{j}}(\eta) \frac{\partial \eta_{k}}{\partial x_{i}} \xi(\eta)+\frac{\partial x_{i}}{\partial \eta_{j}}(\eta) \frac{\partial \eta_{k}}{\partial y_{i}} \xi(\eta)\right)\right)\left(\frac{\partial}{\partial \eta_{k}}\right)-A_{3},
\end{aligned}
$$

so

$$
\begin{aligned}
A_{2}+A_{3}= & \sum_{k=1}^{2 n}\left(\sum_{j=1}^{2 n} a_{j}(\eta) \sum_{i=1}^{n} \frac{\partial}{\partial \eta_{1}}\left(-\frac{\partial y_{i}}{\partial \eta_{j}}(\eta) \frac{\partial \eta_{k}}{\partial x_{i}} \xi(\eta)+\frac{\partial x_{i}}{\partial \eta_{j}}(\eta) \frac{\partial \eta_{k}}{\partial y_{i}} \xi(\eta)\right)\right)\left(\frac{\partial}{\partial \eta_{k}}\right) \\
= & \sum_{k=1}^{2 n} \frac{\partial}{\partial \eta_{1}}\left(\sum_{j=1}^{2 n} a_{j}(\eta) \sum_{i=1}^{n}\left(-\frac{\partial y_{i}}{\partial \eta_{j}}(\eta) \frac{\partial \eta_{k}}{\partial x_{i}} \xi(\eta)+\sum_{i=1}^{n} \frac{\partial x_{i}}{\partial \eta_{j}}(\eta) \frac{\partial \eta_{k}}{\partial y_{i}} \xi(\eta)\right)\right)\left(\frac{\partial}{\partial \eta_{k}}\right) \\
& -\sum_{k=1}^{2 n}\left(\sum_{j=1}^{2 n} \frac{\partial a_{j}(\eta)}{\partial \eta_{1}} \sum_{i=1}^{n}\left(-\frac{\partial y_{i}}{\partial \eta_{j}}(\eta) \frac{\partial \eta_{k}}{\partial x_{i}} \xi(\eta)+\sum_{i=1}^{n} \frac{\partial x_{i}}{\partial \eta_{j}}(\eta) \frac{\partial \eta_{k}}{\partial y_{i}} \xi(\eta)\right)\right)\left(\frac{\partial}{\partial \eta_{k}}\right) .
\end{aligned}
$$

By (3.2) and (3.5) we obtain

$$
A_{2}+A_{3}=[Z, J V](\eta)-J[Z, V](\eta),
$$

and by (3.22), (3.23) and (3.25) it follows that

$$
A=J V(\eta) t(p \circ \pi)(\eta)([Z, J V](\eta)-J[Z, V](\eta))+o(t) .
$$

We will suppose in the sequel of the proof that the restriction of $V$ on $L \cap U$ is a section of $T L \cap J T L$ on $L \cap U$.

Step 5. First order development of $B$ defined in (3.16). 
By (3.6), (3.7) and (3.9) we have

$$
\begin{aligned}
\pi_{* \Phi_{a_{t}}(\eta)}\left(\frac{\partial}{\partial \eta_{k}}\right)_{\Phi_{a_{t}}(\eta)} & =\frac{\partial \varphi}{\partial \eta_{k}}\left(\Phi_{a_{t}}(\eta)\right)\left(\frac{\partial}{\partial \eta_{1}}\right)_{\pi\left(\Phi_{a_{t}}(\eta)\right)}+\left(\frac{\partial}{\partial \eta_{k}}\right)_{\Phi_{a_{t}}(\eta)} \\
& =-\frac{\frac{\partial r}{\partial \eta_{k}}\left(\Phi_{a_{t}}(\eta)\right)}{\frac{\partial r}{\partial \eta_{1}}\left(\Phi_{a_{t}}(\eta)\right)}\left(\frac{\partial}{\partial \eta_{1}}\right)_{\pi\left(\Phi_{a_{t}}(\eta)\right)}+\left(\frac{\partial}{\partial \eta_{k}}\right)_{\pi\left(\Phi_{a_{t}}(\eta)\right)} \\
& =-\frac{\frac{\partial r}{\partial \eta_{k}}(\eta)}{\frac{\partial r}{\partial \eta_{1}}(\eta)}\left(\frac{\partial}{\partial \eta_{1}}\right)_{\pi\left(\Phi_{a_{t}}(\eta)\right)}+\left(\frac{\partial}{\partial \eta_{k}}\right)_{\pi\left(\Phi_{a_{t}}(\eta)\right)}+o(t),
\end{aligned}
$$

so by (3.12)

$$
\begin{aligned}
& \left(\pi_{*}\right)_{\Phi_{a_{t}}(\eta)}\left(J\left(\Phi_{a_{t}}\right)_{* \eta}\left(\sum_{j=1}^{2 n} a_{j}(\eta)\left(\frac{\partial}{\partial \eta_{j}}\right)\right)\right) \\
& =\left(\sum_{j=1}^{2 n} a_{j}(\eta) \sum_{k=1}^{2 n} \sum_{i=1}^{n}-\frac{\partial y_{i}}{\partial \eta_{j}}\left(\Phi_{a_{t}}(\eta)\right) \frac{\partial \eta_{k}}{\partial x_{i}}\left(\xi\left(\Phi_{a_{t}}(\eta)\right)\right)\right. \\
& \left.\quad+\sum_{k=1}^{2 n} \sum_{i=1}^{n} \frac{\partial x_{i}}{\partial \eta_{j}}\left(\left(\Phi_{a_{t}}(\eta)\right)\right) \frac{\partial \eta_{k}}{\partial y_{i}}\left(\xi\left(\Phi_{a_{t}}(\eta)\right)\right)\right) \\
& \quad \times\left(\pi_{*}\right)_{\Phi_{a_{t}}(\eta)}\left(\left(\frac{\partial}{\partial \eta_{k}}\right)_{\Phi_{a_{t}}(\eta)}\right) \\
& \quad+t(p \circ \pi)_{* \eta}\left(\sum_{j=1}^{2 n} a_{j}(\eta)\left(\frac{\partial}{\partial \eta_{j}}\right)\right)\left(\pi_{*}\right)_{\Phi_{a_{t}}(\eta)}\left(J\left(\frac{\partial}{\partial \eta_{1}}\right)_{\Phi_{a_{t}}(\eta)}\right)+o(t) .
\end{aligned}
$$

By (3.9) and (3.19)

$$
\begin{aligned}
\left(\pi_{*}\right)_{\Phi_{a_{t}}(\eta)}\left(J\left(\Phi_{a_{t}}\right)_{* \eta}\left(\sum_{j=1}^{2 n} a_{j}(\eta)\left(\frac{\partial}{\partial \eta_{j}}\right)\right)\right) \\
=\left(\sum_{k=1}^{2 n}\left(\sum_{j=1}^{2 n} a_{j}(\eta)\left(\sum_{i=1}^{n}\left(-\frac{\partial y_{i}}{\partial \eta_{j}} \frac{\partial \eta_{k}}{\partial x_{i}}+\frac{\partial x_{i}}{\partial \eta_{j}} \frac{\partial \eta_{k}}{\partial y_{i}}\right)(\eta)\right)\right)\right) \\
\quad \times \pi_{*} \Phi_{a_{t}}(\eta)\left(\left(\frac{\partial}{\partial \eta_{k}}\right)_{\Phi_{a_{t}}(\eta)}\right)+o(t) \\
=\sum_{k=1}^{2 n} \sum_{j=1}^{2 n} a_{j}(\eta)\left(\sum_{i=1}^{n}\left(-\frac{\partial y_{i}}{\partial \eta_{j}} \frac{\partial \eta_{k}}{\partial x_{i}}+\frac{\partial x_{i}}{\partial \eta_{j}} \frac{\partial \eta_{k}}{\partial y_{i}}\right)(\eta)\right) \\
\quad \times\left(-\frac{\partial r}{\partial \eta_{k}}(\eta)\right. \\
\frac{\partial r}{\partial \eta_{1}}\left(\Phi_{a_{t}}(\eta)\right)
\end{aligned}
$$




$$
\begin{aligned}
= & -\frac{1}{\frac{\partial r}{\partial \eta_{1}}\left(\Phi_{a_{t}}(\eta)\right)}\left(\sum_{k=1}^{2 n}(J V)_{k}(\eta) \frac{\partial r}{\partial \eta_{k}}(\eta)\right)\left(\frac{\partial}{\partial \eta_{1}}\right)_{\pi\left(\Phi_{a_{t}}(\eta)\right)} \\
& +\sum_{k=1}^{2 n}(J V)_{k}(\eta)\left(\frac{\partial}{\partial \eta_{k}}\right)_{\pi\left(\Phi_{a_{t}}(\eta)\right)}+o(t)
\end{aligned}
$$

and by using (3.27) we obtain

$$
\begin{aligned}
\left(\pi_{*}\right)_{\Phi_{a_{t}}}(\eta) & \left(J\left(\Phi_{a_{t}}\right)_{* \eta}\left(\sum_{j=1}^{2 n} a_{j}(\eta)\left(\frac{\partial}{\partial \eta_{j}}\right)\right)\right) \\
= & \sum_{k=1}^{2 n} \sum_{j=1}^{2 n} a_{j}(\eta)\left(\sum_{i=1}^{n}\left(-\frac{\partial y_{i}}{\partial \eta_{j}} \frac{\partial \eta_{k}}{\partial x_{i}}+\frac{\partial x_{i}}{\partial \eta_{j}} \frac{\partial \eta_{k}}{\partial y_{i}}\right)(\eta)\right) \\
\times & \left(-\frac{\partial r}{\partial \eta_{k}}(\eta)\right. \\
& +o(t)\left(\frac{\partial}{\partial \eta_{1}}\left(\Phi_{a_{t}}(\eta)\right)\right. \\
= & -\frac{1}{\frac{\partial r}{\partial \eta_{1}}\left(\Phi_{a_{t}}(\eta)\right)}\left(\sum_{\Phi_{a_{t}}(\eta)}+\left(\frac{\partial}{\partial \eta_{k}}\right)_{\Phi_{a_{t}(\eta)}}(J V)_{k}(\eta) \frac{\partial r}{\partial \eta_{k}}(\eta)\right)_{\left(\frac{\partial}{\partial \eta_{1}}\right)_{\pi\left(\Phi_{a_{t}}(\eta)\right)}} \\
& +\sum_{k=1}^{2 n}(J V)_{k}(\eta)\left(\frac{\partial}{\partial \eta_{k}}\right)_{\pi\left(\Phi_{a_{t}}(\eta)\right)}+o(t) .
\end{aligned}
$$

Since $V$ is a section of $T L \cap J T L$ on $L \cap U$, the first term above vanishes and

$$
\begin{aligned}
& \left(\pi_{*}\right)_{\Phi_{a_{t}}(\eta)}\left(\sum_{k=1}^{2 n}\left(\sum_{j=1}^{2 n} a_{j}(\eta)\left(\sum_{i=1}^{n}\left(-\frac{\partial y_{i}}{\partial \eta_{j}}\left(\Phi_{a_{t}}(\eta)\right) \frac{\partial \eta_{k}}{\partial x_{i}}\left(\xi\left(\Phi_{a_{t}}(\eta)\right)\right)\right)\right)\right)\left(\frac{\partial}{\partial \eta_{k}}\right)_{\Phi_{a_{t}}(\eta)}\right) \\
& =J V+o(t) .
\end{aligned}
$$

It follows that

$$
\begin{aligned}
B & =-t(p \circ \pi)_{* \Phi_{a_{t}}(\eta)}\left(J\left(\Phi_{a_{t}}\right)_{* \eta}\left(\sum_{j=1}^{2 n} a_{j}(\eta)\left(\frac{\partial}{\partial \eta_{j}}\right)\right)\right)\left(\frac{\partial}{\partial \eta_{1}}\right)_{\eta} \\
& =-t\left(p_{*}\right)_{\pi\left(\Phi_{a_{t}}(\eta)\right)}(J V)\left(\frac{\partial}{\partial \eta_{1}}\right)_{\eta}+o(t .)
\end{aligned}
$$

Step 6. First order development of $C$ defined in (3.17).

By (3.5) and (3.13)

$$
\begin{aligned}
& \left(\Phi_{a_{t}}\right)_{*, \Phi_{a_{t}}(\eta)}^{-1}\left(J\left(\frac{\partial}{\partial \eta_{1}}\right)_{\Phi_{a_{t}}(\eta)}\right) \\
& =\sum_{k=1}^{2 n}\left(\sum_{i=1}^{n}-\frac{\partial y_{i}}{\partial \eta_{1}}\left(\Phi_{a_{t}}(\eta)\right) \frac{\partial \eta_{k}}{\partial x_{i}}\left(\xi\left(\Phi_{a_{t}}(\eta)\right)\right)+\frac{\partial x_{i}}{\partial \eta_{1}}\left(\Phi_{a_{t}}(\eta)\right) \frac{\partial \eta_{k}}{\partial y_{i}}\left(\xi\left(\Phi_{a_{t}}(\eta)\right)\right)\right) \\
& \quad \times\left(\frac{\partial}{\partial \eta_{k}}\right)+o(t)
\end{aligned}
$$


and by (3.9), (3.5) and (3.19) it follows that

$$
\begin{aligned}
\left(\Phi_{a_{t}}\right)_{*, \Phi_{a_{t}}(\eta)}^{-1}\left(J\left(\frac{\partial}{\partial \eta_{1}}\right)_{\Phi_{a_{t}}(\eta)}\right)= & \sum_{k=1}^{2 n}\left(\sum_{i=1}^{n}-\frac{\partial y_{i}}{\partial \eta_{1}}(\eta) \frac{\partial \eta_{k}}{\partial x_{i}}(\xi(\eta))\right. \\
& \left.+\frac{\partial x_{i}}{\partial \eta_{1}}(\eta) \frac{\partial \eta_{k}}{\partial y_{i}}(\xi(\eta))\right)\left(\frac{\partial}{\partial \eta_{k}}\right)+o(t) \\
= & J\left(\frac{\partial}{\partial \eta_{1}}\right)_{\eta}+o(t) .
\end{aligned}
$$

which implies that

$$
C=t(p \circ \pi)_{* \eta}\left(\sum_{j=1}^{2 n} a_{j}(\eta)\left(\frac{\partial}{\partial \eta_{j}}\right)\right) J\left(\frac{\partial}{\partial \eta_{1}}\right)_{\eta}+o(t) .
$$

Step 7 End of the proof.

Finally, from (3.14), (3.26), (3.28) and (3.29) we obtain

$$
\begin{aligned}
& \left(\Phi_{a_{t}}\right)_{*, \Phi_{a_{t}}(\eta)}^{-1} J\left(\Phi_{a_{t}}\right)_{*, \eta}(V) \\
& =J V(\eta)+t(p \circ \pi)(\eta)([Z, J V](\eta)-J[Z, V](\eta))-t\left(\left(p_{*}\right)_{\pi\left(\Phi_{a_{t}}(\eta)\right)}(J V)\right) Z(\eta) \\
& \quad+t\left(\left(p_{*}\right)_{\pi\left(\Phi_{a_{t}}(\eta)\right)}(V)\right)(J Z)(\eta)+o(t) .
\end{aligned}
$$

We will prove now Proposition 2:

Proof Since $\alpha_{0}=0$, from (2.5) we have

$$
\begin{aligned}
{\frac{d \alpha_{a_{t}}}{d t \mid t=0}}_{\mid}(V)= & \frac{d}{d t \mid t=0}\left(\left(d_{J_{a_{t}}}^{c} r(X)\right)^{-1} j^{*}\left(d_{J_{a_{t}}}^{c} r\right)-\gamma\right)(V) \\
= & \left.\frac{d}{d t}\right|_{\mid t=0}\left(\left(d_{J_{a_{t}}}^{c} r(X)\right)^{-1}\right) j^{*}\left(d^{c} r\right)(V) \\
& +\left(j^{*} d^{c} r(J Z)\right)^{-1} \frac{d}{d t}{ }_{\mid t=0} j^{*}\left(d_{J_{a_{t}}}^{c} r\right)(V) .
\end{aligned}
$$

Since $j^{*}\left(d^{c} r\right)(V)=\gamma(V)=0$ and $j^{*} d^{c} r(J Z)=\gamma(X)=1$ we obtain

$$
{\frac{d \alpha_{a_{t}}}{d t}}_{\mid t=0}(V)=\frac{d}{d t}_{\mid t=0} j^{*}\left(d_{J_{a_{t}}}^{c} r\right)(V) .
$$

But

$$
\begin{aligned}
&{\left.\frac{d}{d t}\right|_{t=0} j^{*}\left(d_{J_{a_{t}}}^{c} r\right)(V)}=\frac{d}{d t \mid t=0} \\
&=-(d r) \frac{d}{d t}_{\mid t=0}\left(J_{a_{t}} d r\right)(V) \\
&=-(d r) \frac{d}{d t}_{\mid t=0}\left(\left(\left(\Phi_{a_{t}}^{-1}\right)_{*} \circ J \circ\left(\Phi_{a_{t}}\right)_{*}\right)(V)\right),
\end{aligned}
$$


and by Lemma 4 it follows that

$$
{\frac{d \alpha_{a_{t}}}{d t}{ }_{\mid t=0}}(V)=-(d r)\left(p([Z, J V]-J[Z, V])-\left(p_{*}(J V)\right) Z+\left(p_{*}(V)\right) J Z\right) .
$$

Since

$$
0=d d r(Z, J V)=Z(d r(J V))-J V(d r(Z))-d r[Z, J V]
$$

and $d r(Z)=1$, we have

$$
d r[Z, J V]=Z(d r(J V)) .
$$

Similarly, since

$$
\begin{aligned}
d d^{c} r(Z, V) & =Z\left(d^{c} r(V)\right)-V\left(d^{c} r(Z)\right)-d^{c} r([Z, V]) \\
& =-Z(d r(J V))+V(J(d r(Z)))+d r(J[Z, V]) \\
& =-Z(d r(J V))+d r(J[Z, V]),
\end{aligned}
$$

we have

$$
d r(J[Z, V])=d d^{c} r(Z, V)+Z(d r(J V)) .
$$

By replacing $d r[Z, J V]$ and $d r(J[Z, V])$ from (3.31) and (3.33) in (3.30), since $d r(J Z)=d r(X)=0$, it follows that

$$
\begin{aligned}
{\frac{d \alpha_{a_{t}}}{d t}{ }_{\mid t=0}(V)}=-p Z(d r(J V))+p\left(d d^{c} r(Z, V)+Z(J d r(V))\right)+J V(p) \\
=p d d^{c} r(Z, V)+J V(p)=p d d^{c} r(J Z, J V)-d^{c} p(V) .
\end{aligned}
$$

Since

$$
d d^{c} r(J Z, J V)=\iota_{J Z} d d^{c} r(J V)=\iota_{X} J d d^{c} r(V),
$$

we obtain

$$
{\frac{d \alpha_{a_{t}}}{d t}}_{\mid t=0}(V)=p \iota_{X} J d d^{c} r(V)-d^{c} p(V)
$$

Proposition 3 Let $L$ be a Levi flat hypersurface in $M$ and $\left(a_{t}\right)_{t \in]-\varepsilon, \varepsilon[}$ a differentiable family in $C^{\infty}(L)$ which defines a Levi flat deformation of $L$. Set $p=\frac{d a_{t}}{d t}{ }_{\mid t=0}$. Then, for each vector field $V$ on $M$ such that the restriction of $V$ to $L$ is a section of $T L \cap J T L$, we have

$$
{\frac{d \gamma_{t}}{d t}}_{\mid t=0}(V)=-p J \iota_{X} d d^{c} r(V)+d^{c} p(V) .
$$

Proof For every $x \in L$ and $V \in T_{x} L$ we have

$$
\begin{aligned}
\gamma_{a_{t}}(x)(V) & =\Phi_{a_{t}}^{*}\left(\eta_{a_{t}}\right)(x)(V) \\
& =\eta_{a_{t}}\left(\Phi_{a_{t}}(x)\right)\left(\left(\Phi_{a_{t}}\right)_{*, x}(V)\right)=j_{a_{t}}^{*}\left(d^{c} r_{a_{t}}\right)\left(\Phi_{a_{t}}(x)\right)\left(\left(\Phi_{a_{t}}\right)_{*, x}(V)\right) \\
& =\left(d^{c} r_{a_{t}}\right)\left(j_{a_{t}}\left(\Phi_{a_{t}}(x)\right)\right)\left(j_{a_{t}}\right)_{*}\left(\left(\Phi_{a_{t}}\right)_{*, x}(V)\right) \\
& =\left(d^{c} r_{a_{t}}\right)\left(\Phi_{a_{t}}(x)\right)\left(\left(\Phi_{a_{t}}\right)_{*, x}(V)\right) \\
& =\left(-J d r+J d\left(a_{t} \circ \pi\right)\right)\left(\Phi_{a_{t}}(x)\right)\left(\left(\Phi_{a_{t}}\right)_{*, x}(V)\right) \\
& =-J d r\left(\Phi_{a_{t}}(x)\right)\left(\left(\Phi_{a_{t}}\right)_{*, x}(V)\right)+J d\left(a_{t} \circ \pi\right)\left(\Phi_{a_{t}}(x)\right)\left(\Phi_{a_{t}}\right)_{*, x}(V)
\end{aligned}
$$




$$
=-d r\left(\Phi_{a_{t}}(x)\right)\left(J\left(\Phi_{a_{t}}\right)_{*, x}(V)\right)+d\left(a_{t} \circ \pi\right)\left(\Phi_{a_{t}}(x)\right)\left(J\left(\Phi_{a_{t}}\right)_{*, x}(V)\right) .
$$

We will consider the coordinates $\eta$ in a neighborhood of $x$ defined in the first step of the proof of Lemma 4 such that $Z=\frac{\partial}{\partial \eta_{1}}$ on $U$.

Step 1. First order development of $d r\left(\Phi_{a_{t}}(x)\right)\left(J\left(\Phi_{a_{t}}\right)_{*, x}(V)\right)$.

Set $V=\sum_{j=1}^{2 n} a_{j}(\eta)\left(\frac{\partial}{\partial \eta_{j}}\right)$. Since $d r\left(\Phi_{a_{t}}(\eta)\right) \cdot(J Z)\left(\Phi_{a_{t}}(\eta)\right)=0$, by (3.12) we have

$$
\begin{aligned}
d r & \left(\Phi_{a_{t}}(\eta)\right)\left(J\left(\Phi_{a_{t}}\right)_{*, \eta}(V)\right)=\sum_{k=1}^{2 n} \sum_{j=1}^{2 n} a_{j}(\eta) \\
& \times\left(\sum_{i=1}^{n}\left(-\frac{\partial y_{i}}{\partial \eta_{j}}\left(\Phi_{a_{t}}(\eta)\right) \frac{\partial \eta_{k}}{\partial x_{i}}\left(\xi\left(\Phi_{a_{t}}(\eta)\right)\right)+\frac{\partial x_{i}}{\partial \eta_{j}}\left(\Phi_{a_{t}}(\eta)\right) \frac{\partial \eta_{k}}{\partial y_{i}}\left(\xi\left(\Phi_{a_{t}}(\eta)\right)\right)\right)\right) \\
& \times \frac{\partial r}{\partial \eta_{k}}\left(\Phi_{a_{t}}(\eta)\right)+o(t) .
\end{aligned}
$$

But by using (3.9), (3.20) and (3.21) we obtain

$$
\begin{aligned}
- & \frac{\partial y_{i}}{\partial \eta_{j}}\left(\Phi_{a_{t}}(\eta)\right) \frac{\partial \eta_{k}}{\partial x_{i}}\left(\xi\left(\Phi_{a_{t}}(\eta)\right)\right) \\
= & \left(\sum_{i=1}^{n}-\frac{\partial y_{i}}{\partial \eta_{j}}\left(\eta+t(p \circ \pi)(\eta) e_{1}\right)\right) \\
& \times\left(\frac{\partial \eta_{k}}{\partial x_{i}}(\xi(\eta))+t(p \circ \pi)(\eta) \frac{\partial}{\partial \eta_{1}}\left(\frac{\partial \eta_{k}}{\partial x_{i}} \xi(\eta)\right)\right)+o(t) \\
= & \left(\sum_{i=1}^{n}\left(-\frac{\partial y_{i}}{\partial \eta_{j}}(\eta)-t(p \circ \pi) \frac{\partial}{\partial \eta_{1}}\left(\frac{\partial y_{i}}{\partial \eta_{j}}\right)(\eta)\right)\right) \\
& \times\left(\frac{\partial \eta_{k}}{\partial x_{i}}(\xi(\eta))+t(p \circ \pi)(\eta) \frac{\partial}{\partial \eta_{1}}\left(\frac{\partial \eta_{k}}{\partial x_{i}} \xi(\eta)\right)\right)+o(t)
\end{aligned}
$$

and

$$
\begin{aligned}
& \frac{\partial x_{i}}{\partial \eta_{j}}\left(\Phi_{a_{t}}(\eta)\right) \frac{\partial \eta_{k}}{\partial y_{i}}\left(\xi\left(\Phi_{a_{t}}(\eta)\right)\right) \\
& =\frac{\partial x_{i}}{\partial \eta_{j}}\left(\eta+t(p \circ \pi) e_{1}\right)\left(\frac{\partial \eta_{k}}{\partial y_{i}}(\xi(\eta))+t(p \circ \pi)(\eta) \frac{\partial}{\partial \eta_{1}}\left(\frac{\partial \eta_{k}}{\partial y_{i}} \xi(\eta)\right)\right)+o(t) \\
& =\left(\frac{\partial x_{i}}{\partial \eta_{j}}(\eta)+t(p \circ \pi) \frac{\partial}{\partial \eta_{1}}\left(\frac{\partial x_{i}}{\partial \eta_{j}}\right)(\eta)\right) \\
& \quad \times\left(\frac{\partial \eta_{k}}{\partial y_{i}}(\xi(\eta))+t(p \circ \pi)(\eta) \frac{\partial}{\partial \eta_{1}}\left(\frac{\partial \eta_{k}}{\partial y_{i}} \xi(\eta)\right)\right)+o(t),
\end{aligned}
$$

so by replacing (3.37) and (3.38) in (3.36) it follows that

$$
\begin{aligned}
d r & \left(\Phi_{a_{t}}(\eta)\right)\left(J\left(\Phi_{a_{t}}\right)_{*, \eta}(V)\right) \\
& =\sum_{k=1}^{2 n} \sum_{j=1}^{2 n} a_{j}(\eta)\left(\sum_{i=1}^{n}-\frac{\partial y_{i}}{\partial \eta_{j}}(\eta) \frac{\partial \eta_{k}}{\partial x_{i}}(\xi(\eta))+\frac{\partial x_{i}}{\partial \eta_{j}}(\eta) \frac{\partial \eta_{k}}{\partial y_{i}}(\xi(\eta))\right) \frac{\partial r}{\partial \eta_{k}}\left(\Phi_{a_{t}}(\eta)\right)
\end{aligned}
$$




$$
\begin{aligned}
& +t(p \circ \pi)(\eta) \sum_{k=1}^{2 n}\left(\sum_{j=1}^{2 n} a_{j}(\eta) \sum_{i=1}^{n}\left(-\frac{\partial y_{i}}{\partial \eta_{j}}(\eta)\right) \frac{\partial}{\partial \eta_{1}}\left(\frac{\partial \eta_{k}}{\partial x_{i}} \xi(\eta)\right)+\frac{\partial x_{i}}{\partial \eta_{j}}(\eta) \frac{\partial}{\partial \eta_{1}}\left(\frac{\partial \eta_{k}}{\partial y_{i}} \xi(\eta)\right)\right) \\
& \times \frac{\partial r}{\partial \eta_{k}}\left(\Phi_{a_{t}}(\eta)\right) \\
& +t(p \circ \pi)(\eta) \sum_{k=1}^{2 n} \sum_{j=1}^{2 n} a_{j}(\eta)\left(\sum_{i=1}^{n}-\frac{\partial}{\partial \eta_{1}}\left(\frac{\partial y_{i}}{\partial \eta_{j}}\right)(\eta) \frac{\partial \eta_{k}}{\partial x_{i}}(\xi(\eta))+\frac{\partial}{\partial \eta_{1}}\left(\frac{\partial x_{i}}{\partial \eta_{j}}\right)(\eta) \frac{\partial \eta_{k}}{\partial y_{i}}(\xi(\eta))\right) \\
& \times \frac{\partial r}{\partial \eta_{k}}\left(\Phi_{a_{t}}(\eta)\right)+o(t) .
\end{aligned}
$$

By (3.5)

$$
\begin{aligned}
& \sum_{k=1}^{2 n} \sum_{j=1}^{2 n} a_{j}(\eta)\left(\sum_{i=1}^{n}-\frac{\partial y_{i}}{\partial \eta_{j}}(\eta) \frac{\partial \eta_{k}}{\partial x_{i}}(\xi(\eta))+\frac{\partial x_{i}}{\partial \eta_{j}}(\eta) \frac{\partial \eta_{k}}{\partial y_{i}}(\xi(\eta))\right) \frac{\partial r}{\partial \eta_{k}}\left(\Phi_{a_{t}}(\eta)\right) \\
& \quad=d r\left(\Phi_{a_{t}}(\eta)\right) \cdot J V(\eta)
\end{aligned}
$$

so

$$
\begin{aligned}
d r & \left(\Phi_{a_{t}}(\eta)\right)\left(J\left(\Phi_{a_{t}}\right)_{*, \eta}(V)\right) \\
= & d r\left(\Phi_{a_{t}}(\eta)\right) \cdot J V(\eta) \\
& +t(p \circ \pi)(\eta) \sum_{k=1}^{2 n}\left(\sum_{j=1}^{2 n} a_{j}(\eta) \sum_{i=1}^{n}\left(-\frac{\partial y_{i}}{\partial \eta_{j}}(\eta)\right) \frac{\partial}{\partial \eta_{1}}\left(\frac{\partial \eta_{k}}{\partial x_{i}} \xi(\eta)\right)+\frac{\partial x_{i}}{\partial \eta_{j}}(\eta) \frac{\partial}{\partial \eta_{1}}\left(\frac{\partial \eta_{k}}{\partial y_{i}} \xi(\eta)\right)\right) \\
& \times \frac{\partial r}{\partial \eta_{k}}\left(\Phi_{a_{t}}(\eta)\right) \\
& +t(p \circ \pi)(\eta) \sum_{k=1}^{2 n} \sum_{j=1}^{2 n} a_{j}(\eta)\left(\sum_{i=1}^{n}-\frac{\partial}{\partial \eta_{1}}\left(\frac{\partial y_{i}}{\partial \eta_{j}}\right)(\eta) \frac{\partial \eta_{k}}{\partial x_{i}}(\xi(\eta))+\frac{\partial}{\partial \eta_{1}}\left(\frac{\partial x_{i}}{\partial \eta_{j}}\right)(\eta) \frac{\partial \eta_{k}}{\partial y_{i}}(\xi(\eta))\right) \\
& \times \frac{\partial r}{\partial \eta_{k}}\left(\Phi_{a_{t}}(\eta)\right)+o(t) .
\end{aligned}
$$

We will now compute the first order development of $d r\left(\Phi_{a_{t}}(\eta)\right) \cdot J V(\eta)$.

By (3.9) we have

$$
\begin{aligned}
& \frac{\partial r}{\partial \eta_{k}}\left(\Phi_{a_{t}}(\eta)\right)=\frac{\partial r}{\partial \eta_{k}}\left(\eta+t p(\pi(\eta)) e_{1}+o(t)\right) \\
& \quad=\frac{\partial r}{\partial \eta_{k}}(\eta)+t p(\pi(\eta)) \frac{\partial}{\partial \eta_{1}}\left(\frac{\partial r}{\partial \eta_{k}}\right)(\eta)+o(t),
\end{aligned}
$$

and it follows that

$$
\begin{aligned}
d r & \left(\Phi_{a_{t}}(\eta)\right) \cdot J V(\eta) \\
= & \sum_{k=1}^{2 n} \frac{\partial r}{\partial \eta_{k}}\left(\Phi_{a_{t}}(\eta)\right) \sum_{j=1}^{2 n} a_{j}(\eta)\left(\sum_{i=1}^{n}-\frac{\partial y_{i}}{\partial \eta_{j}}(\eta) \frac{\partial \eta_{k}}{\partial x_{i}}(\xi(\eta))+\frac{\partial x_{i}}{\partial \eta_{j}}(\eta) \frac{\partial \eta_{k}}{\partial y_{i}}(\xi(\eta))\right) \\
= & \sum_{k=1}^{2 n}\left(\frac{\partial r}{\partial \eta_{k}}(\eta)+t p(\pi(\eta)) \frac{\partial}{\partial \eta_{1}}\left(\frac{\partial r}{\partial \eta_{k}}\right)(\eta)\right) \\
& \times \sum_{j=1}^{2 n} a_{j}(\eta)\left(\sum_{i=1}^{n}-\frac{\partial y_{i}}{\partial \eta_{j}}(\eta) \frac{\partial \eta_{k}}{\partial x_{i}}(\xi(\eta))+\frac{\partial x_{i}}{\partial \eta_{j}}(\eta) \frac{\partial \eta_{k}}{\partial y_{i}}(\xi(\eta))\right)+o(t)
\end{aligned}
$$




$$
\begin{aligned}
= & \sum_{k=1}^{2 n} \sum_{j=1}^{2 n} a_{j}(\eta)\left(\sum_{i=1}^{n}-\frac{\partial y_{i}}{\partial \eta_{j}}(\eta) \frac{\partial \eta_{k}}{\partial x_{i}}(\xi(\eta))+\frac{\partial x_{i}}{\partial \eta_{j}}(\eta) \frac{\partial \eta_{k}}{\partial y_{i}}(\xi(\eta))\right) \frac{\partial r}{\partial \eta_{k}}(\eta) \\
& +t p(\pi(\eta)) \sum_{k=1}^{2 n}\left(\sum _ { j = 1 } ^ { 2 n } a _ { j } ( \eta ) \left(\sum_{i=1}^{n}-\frac{\partial y_{i}}{\partial \eta_{j}}(\eta) \frac{\partial \eta_{k}}{\partial x_{i}}(\xi(\eta))\right.\right. \\
& \left.\left.+\frac{\partial x_{i}}{\partial \eta_{j}}(\eta) \frac{\partial \eta_{k}}{\partial y_{i}}(\xi(\eta))\right)\right) \frac{\partial}{\partial \eta_{1}}\left(\frac{\partial r}{\partial \eta_{k}}\right)(\eta)+o(t) .
\end{aligned}
$$

Since $J V$ is tangent to $L$, (3.5) implies that

$$
\begin{aligned}
& \sum_{k=1}^{2 n} \sum_{j=1}^{2 n} a_{j}(\eta)\left(\sum_{i=1}^{n}-\frac{\partial y_{i}}{\partial \eta_{j}}(\eta) \frac{\partial \eta_{k}}{\partial x_{i}}(\xi(\eta))+\frac{\partial x_{i}}{\partial \eta_{j}}(\eta) \frac{\partial \eta_{k}}{\partial y_{i}}(\xi(\eta))\right) \frac{\partial r}{\partial \eta_{k}}(\eta) \\
& \quad=d r(\eta) \cdot J V(\eta)=0
\end{aligned}
$$

and therefore

$$
\begin{aligned}
d r & \left(\Phi_{a_{t}}(\eta)\right) \cdot J V(\eta) \\
= & t p(\pi(\eta)) \sum_{k=1}^{2 n}\left(\sum _ { j = 1 } ^ { 2 n } a _ { j } ( \eta ) \left(\sum_{i=1}^{n}-\frac{\partial y_{i}}{\partial \eta_{j}}(\eta) \frac{\partial \eta_{k}}{\partial x_{i}}(\xi(\eta))\right.\right. \\
& \left.\left.+\frac{\partial x_{i}}{\partial \eta_{j}}(\eta) \frac{\partial \eta_{k}}{\partial y_{i}}(\xi(\eta))\right)\right) \cdot \frac{\partial}{\partial \eta_{1}}\left(\frac{\partial r}{\partial \eta_{k}}\right)(\eta)+o(t) .
\end{aligned}
$$

To end the Step 1, we replace now (3.40) in (3.39) and we obtain

$$
\begin{aligned}
d r\left(\Phi_{a_{t}}(\eta)\right)\left(J\left(\Phi_{a_{t}}\right)_{*, \eta}(V)\right) \\
=t(p \circ \pi)(\eta) \sum_{k=1}^{2 n}\left(\sum _ { j = 1 } ^ { 2 n } a _ { j } ( \eta ) \left(\sum_{i=1}^{n}-\frac{\partial y_{i}}{\partial \eta_{j}}(\eta) \frac{\partial \eta_{k}}{\partial x_{i}}(\xi(\eta))\right.\right. \\
\left.\left.+\frac{\partial x_{i}}{\partial \eta_{j}}(\eta) \frac{\partial \eta_{k}}{\partial y_{i}}(\xi(\eta))\right)\right) \frac{\partial}{\partial \eta_{1}}\left(\frac{\partial r}{\partial \eta_{k}}\right)(\eta) \\
+t(p \circ \pi)(\eta) \sum_{k=1}^{2 n}\left(\sum_{j=1}^{2 n} a_{j}(\eta) \sum_{i=1}^{n}\left(-\frac{\partial y_{i}}{\partial \eta_{j}}(\eta)\right) \frac{\partial}{\partial \eta_{1}}\left(\frac{\partial \eta_{k}}{\partial x_{i}} \xi(\eta)\right)\right. \\
\left.+\frac{\partial x_{i}}{\partial \eta_{j}}(\eta) \frac{\partial}{\partial \eta_{1}}\left(\frac{\partial \eta_{k}}{\partial y_{i}} \xi(\eta)\right)\right) \frac{\partial r}{\partial \eta_{k}}(\eta) \\
+t(p \circ \pi)(\eta) \sum_{k=1}^{2 n} \sum_{j=1}^{2 n} a_{j}(\eta)\left(\sum_{i=1}^{n}-\frac{\partial}{\partial \eta_{1}}\left(\frac{\partial y_{i}}{\partial \eta_{j}}\right)(\eta) \frac{\partial \eta_{k}}{\partial x_{i}}(\xi(\eta))\right. \\
\left.+\frac{\partial}{\partial \eta_{1}}\left(\frac{\partial x_{i}}{\partial \eta_{j}}\right)(\eta) \frac{\partial \eta_{k}}{\partial y_{i}}(\xi(\eta))\right) \frac{\partial r}{\partial \eta_{k}}(\eta)+o(t) \\
=t p(\pi(\eta)) \sum_{k=1}^{2 n}\left(\sum _ { j = 1 } ^ { 2 n } a _ { j } ( \eta ) \left(\sum_{i=1}^{n}-\frac{\partial y_{i}}{\partial \eta_{j}}(\eta) \frac{\partial \eta_{k}}{\partial x_{i}}(\xi(\eta))\right.\right.
\end{aligned}
$$




$$
\begin{aligned}
& \left.\left.+\frac{\partial x_{i}}{\partial \eta_{j}}(\eta) \frac{\partial \eta_{k}}{\partial y_{i}}(\xi(\eta))\right)\right) \frac{\partial}{\partial \eta_{1}}\left(\frac{\partial r}{\partial \eta_{k}}\right)(\eta) \\
& +t(p \circ \pi)(\eta)([Z, J V]-J[Z, V])(r)(\eta) .
\end{aligned}
$$

But

$$
\begin{aligned}
\sum_{k=1}^{2 n} & \frac{\partial}{\partial \eta_{1}}\left(\frac{\partial r}{\partial \eta_{k}}\right)(\eta)\left(\sum_{j=1}^{2 n} a_{j}(\eta)\left(\sum_{i=1}^{n}-\frac{\partial y_{i}}{\partial \eta_{j}}(\eta) \frac{\partial \eta_{k}}{\partial x_{i}}(\xi(\eta))+\frac{\partial x_{i}}{\partial \eta_{j}}(\eta) \frac{\partial \eta_{k}}{\partial y_{i}}(\xi(\eta))\right)\right) \\
= & \sum_{k=1}^{2 n} \frac{\partial}{\partial \eta_{1}}\left[\left(\frac{\partial r}{\partial \eta_{k}}\right)(\eta)\left(\sum_{j=1}^{2 n} a_{j}(\eta)\left(\sum_{i=1}^{n}-\frac{\partial y_{i}}{\partial \eta_{j}}(\eta) \frac{\partial \eta_{k}}{\partial x_{i}}(\xi(\eta))+\frac{\partial x_{i}}{\partial \eta_{j}}(\eta) \frac{\partial \eta_{k}}{\partial y_{i}}(\xi(\eta))\right)\right)\right] \\
& -\sum_{k=1}^{2 n} \frac{\partial}{\partial \eta_{1}}\left(\sum_{j=1}^{2 n} a_{j}(\eta)\left(\sum_{i=1}^{n}-\frac{\partial y_{i}}{\partial \eta_{j}}(\eta) \frac{\partial \eta_{k}}{\partial x_{i}}(\xi(\eta))+\frac{\partial x_{i}}{\partial \eta_{j}}(\eta) \frac{\partial \eta_{k}}{\partial y_{i}}(\xi(\eta))\right)\right)\left(\frac{\partial r}{\partial \eta_{k}}\right)(\eta) \\
= & Z(d r \cdot J V)(\eta)-[Z, J V](r)(\eta),
\end{aligned}
$$

and it follows that

$$
\begin{aligned}
d r\left(\Phi_{a_{t}}(\eta)\right)\left(J\left(\Phi_{a_{t}}\right)_{*, \eta}(V)\right)= & t(p \circ \pi)(Z(d r \cdot J V)-[Z, J V](r)(\eta) \\
& +([Z, J V]-J[Z, V])(r))(\eta) \\
= & t(p \circ \pi)(Z(d r \cdot J V)-J[Z, V](r))(\eta) .
\end{aligned}
$$

Step 2. First order development of $d\left(a_{t} \circ \pi\right)\left(\Phi_{a_{t}}(\eta)\right)\left(J\left(\Phi_{a_{t}}\right)_{*, \eta}(V)\right)$.

We have

$$
d\left(a_{t} \circ \pi\right)\left(\Phi_{a_{t}}(\eta)\right)\left(J\left(\Phi_{a_{t}}\right)_{*, \eta}(V)\right)=\sum_{k=1}^{2 n} \frac{\partial\left(a_{t} \circ \pi\right)}{\partial \eta_{k}}\left(\Phi_{a_{t}}(\eta)\right)\left(J\left(\Phi_{a_{t}}\right)_{*, \eta}(V)\right)_{k} .
$$

Since (3.9) implies

$$
\begin{aligned}
& \frac{\partial\left(a_{t} \circ \pi\right)}{\partial \eta_{k}}\left(\Phi_{a_{t}}(\eta)\right)=\frac{\partial(t(p \circ \pi)+o(t))}{\partial \eta_{k}}\left(\eta+t p(\pi(\eta)) e_{1}+o(t)\right) \\
& =t \frac{\partial(p \circ \pi)}{\partial \eta_{k}}(\eta)+o(t)
\end{aligned}
$$

by (3.12) it follows that

$$
\begin{aligned}
& d\left(a_{t} \circ \pi\right)\left(\Phi_{a_{t}}(\eta)\right)\left(J\left(\Phi_{a_{t}}\right)_{*, \eta}(V)\right) \\
& =t \sum_{k=1}^{2 n} \sum_{j=1}^{2 n} a_{j}(\eta) \sum_{i=1}^{n}\left(-\frac{\partial y_{i}}{\partial \eta_{j}}\left(\Phi_{a_{t}}(\eta)\right) \frac{\partial \eta_{k}}{\partial x_{i}}\left(\xi\left(\Phi_{a_{t}}(\eta)\right)\right)\right. \\
& \left.\quad+\frac{\partial x_{i}}{\partial \eta_{j}}\left(\Phi_{a_{t}}(\eta)\right) \frac{\partial \eta_{k}}{\partial y_{i}}\left(\xi\left(\Phi_{a_{t}}(\eta)\right)\right)\right) \frac{\partial(p \circ \pi)}{\partial \eta_{k}}(\eta)+o(t)
\end{aligned}
$$

and by using (3.9) and (3.19) we obtain

$$
\begin{aligned}
& d\left(a_{t} \circ \pi\right)\left(\Phi_{a_{t}}(\eta)\right)\left(J\left(\Phi_{a_{t}}\right)_{*, \eta}(V)\right) \\
& =t \sum_{k=1}^{2 n} \sum_{j=1}^{2 n} a_{j}(\eta)\left(\sum_{i=1}^{n}-\frac{\partial y_{i}}{\partial \eta_{j}}\left(\eta+t(p \circ \pi) e_{1}\right)\right)
\end{aligned}
$$




$$
\begin{aligned}
& \times\left(\frac{\partial \eta_{k}}{\partial x_{i}}(\xi(\eta))+t(p \circ \pi)(\eta) \frac{\partial}{\partial \eta_{1}}\left(\frac{\partial \eta_{k}}{\partial x_{i}} \xi(\eta)\right)\right) \frac{\partial(p \circ \pi)}{\partial \eta_{k}}(\eta)+t \sum_{k=1}^{2 n} \sum_{j=1}^{2 n} a_{j}(\eta) \\
& \times\left(\sum_{i=1}^{n} \frac{\partial x_{i}}{\partial \eta_{j}}\left(\eta+t(p \circ \pi) e_{1}\right)\left(\frac{\partial \eta_{k}}{\partial y_{i}}(\xi(\eta))+t(p \circ \pi)(\eta) \frac{\partial}{\partial \eta_{1}}\left(\frac{\partial \eta_{k}}{\partial y_{i}} \xi(\eta)\right)\right)\right) \\
& \times \frac{\partial(p \circ \pi)}{\partial \eta_{k}}(\eta)+o(t) \\
& =t \sum_{k=1}^{2 n} \sum_{j=1}^{2 n} a_{j}(\eta)\left(-\frac{\partial y_{i}}{\partial \eta_{j}}(\eta) \frac{\partial \eta_{k}}{\partial x_{i}}(\xi(\eta))+\frac{\partial x_{i}}{\partial \eta_{j}}(\eta) \frac{\partial \eta_{k}}{\partial y_{i}}(\xi(\eta))\right) \frac{\partial(p \circ \pi)}{\partial \eta_{k}}(\eta)+o(t) \\
& =t d p(\eta) \cdot J V(\eta)+o(t) .
\end{aligned}
$$

Step 3. End of the proof.

From (3.35), (3.41) and (3.43) we have

$$
\begin{aligned}
&{\frac{d \gamma a_{t}}{d t}{ }_{\mid t=0}(V)}=p(Z(d r \cdot J V)-J[Z, V](r))+J V(p) \\
&=-p Z\left(d^{c} r(V)\right)-p J[Z, V](r)+J V(p) .
\end{aligned}
$$

By (3.32)

$$
d d^{c} r(Z, V)=-Z(d r(J V))+d r(J[Z, V])=Z\left(d^{c} r(V)\right)-d^{c} r([Z, V]),
$$

and by replacing in( 3.44$)$ we obtain

$$
\begin{aligned}
&{\left.\frac{d \gamma_{a_{t}}}{d t}\right|_{\mid t=0}(V)=}-p\left(d d^{c} r(Z, V)+d^{c} r([Z, V])\right)-p J[Z, V](r)+J V(p) \\
&=-p d d^{c} r(J Z, J V)+p d r(J[Z, V])-p d r(J[Z, V])+d p(J V) \\
&-p \iota_{J} Z d d^{c} r(J V)-J d p(V) \\
&=-p J \iota_{X} d d^{c} r(V)+d^{c} p(V),
\end{aligned}
$$

and the Proposition 3 is proved.

Corollary 1 Under the notations and hypothesis of the previous propositions,

$$
{\frac{d \gamma_{a_{t}}}{d t}}_{\mid t=0}=-{\frac{d \alpha_{a_{t}}}{d t}}_{\mid t=0} .
$$

Remark 2 By using Proposition 2, it was proved in [1] that

$$
\delta{\frac{d a_{t}}{d t}}_{\mid t=0}=-\delta \delta^{c} p=0,
$$

or equivalently

$$
d_{b} d_{b}^{c} p-d_{b} p \wedge J \mathfrak{b}-d_{b}^{c} p \wedge \mathfrak{b}=p\left(J d_{b}^{c} \mathfrak{b}+\mathfrak{b} \wedge J \mathfrak{b}\right),
$$

where $\mathfrak{b}=\iota_{X} d \gamma$. From Corollary 1 it follows that the same equations are also obtained by means of $\gamma_{a(t)}$. 


\section{References}

1. de Bartolomeis, P., Iordan, A.: Deformations of Levi flat hypersurfaces in complex manifolds. Ann. Scient. Éc. Norm. Sup. 48(2), 281-311 (2015)

2. de Bartolomeis, P., Iordan, A.: Maurer-Cartan equation in the DGLA of graded derivations, (2015), Preprint arXiv: 1506.06732

3. de Bartolomeis, P., Iordan, A.: On the obstruction of the deformation theory in the DGLA of graded derivations, Complex and Symplectic Geometry (D. Angella, C. Medori and A. Tomassini, ed.), vol. 21, Springer Indam Series, pp. 95-105 (2017)

4. Frölicher, A., Nijenhuis, A.: Theory of vector valued differential forms. Part I. Derivations of the graded ring of differential forms. Indag. Math. 18, 338-359 (1956)

5. Godbillon, C.: Feuilletages: Etudes géométriques. Progress in Mathematics, Birkhäuser, Basel (1991)

6. Kodaira, K.: On deformations of some complex pseudo-group structures. Ann. Math. 71, 224-302 (1960)

7. Kodaira, K., Spencer, D.: Multifoliate structures. Ann. Math. 74(1), 52-100 (1961)

8. Michor, P.W.: Topics in differential geometry. AMS, Providence (2008)

9. Spencer, D.C.: Some remarks on perturbation of structure, Analytic Functions, pp. 67-87. Princeton University Press, Princeton, NJ (1960) 